Humans First: Why people value animals less than humans

This manuscript has not yet been published at a peer-reviewed journal.

Lucius Caviola $^{1}$, Stefan Schubert ${ }^{2}$, Guy Kahane ${ }^{2 *}$, Nadira S., Faber ${ }^{2,3 *}$

${ }^{1}$ Department of Psychology, Harvard University

${ }^{2}$ Uehiro Centre for Practical Ethics, University of Oxford

${ }^{2}$ College of Life and Environmental Sciences, University of Exeter

* These authors share senior authorship

Acknowledgments: We thank Oscar Horta for his helpful comments. This research was funded by the University of Oxford and the Janggen-Poehn-Foundation. 


\begin{abstract}
People routinely give humans moral priority over other animals. Is such moral anthropocentrism based in perceived differences in mental capacity between humans and nonhumans or merely because humans favor other members of their own species? We investigated this question in six studies $(\mathrm{N}=2,217)$. We found that most participants prioritized humans over animals even when the animals were described as having equal or more advanced mental capacities than the humans. This applied to both mental capacity at the level of specific individuals (Studies 1a-b) and at the level typical for the respective species (Study 2). The key driver behind moral anthropocentrism was thus mere species-membership (speciesism). However, all else equal, participants still gave more moral weight to individuals with higher mental capacities (individual mental capacity principle), suggesting that the belief that humans have higher mental capacities than animals is part of the reason that they give humans moral priority. Notably, participants found mental capacity more important for animals than for humans - a tendency which can itself be regarded speciesist. We also explored possible subfactors driving speciesism. We found that many participants judged that all individuals (not only humans) should prioritize members of their own species over members of other species (species-relativism; Studies 3a-b). However, some participants also exhibited a tendency to see humans as having superior value in an absolute sense (pro-human species-absolutism, Studies 3-4). Overall, our work demonstrates that speciesism plays a central role in explaining moral anthropocentrism and may be itself divided in multiple sub-factors.
\end{abstract}

Keywords: animals, speciesism, anthropocentrism, moral judgments 


\section{Humans first: Why people value animals less than humans}

Imagine someone bravely entering a burning building to look for survivors. How would you feel if they came out holding a cat, when they could have just as easily saved a fellow human? Most of us would find such a choice unintelligible. It is just obvious that humans should come first. We call this view moral anthropocentrism: the view that humans should be given moral priority over others. In the world around us, this widely held view manifests itself primarily in the ways that we treat non-human animals. The fridges of many of us are laden with cuts of meat. But we would be utterly horrified by the very idea of tasting a morsel of human meat, let alone farming humans for food. And we routinely subject animals to painful experiments, or regard them as property, things that we would never dream of doing to humans.

Moral philosophers debate whether moral anthropocentrism, and the practices it supports, are justified. Some argue that downgrading animals in this way is no more than speciesism (Singer, 1975) — a prejudice against those who do not belong to our own species that is as indefensible as racism and sexism. Those who attempt to uphold moral anthropocentrism attempt to identify features of humans that would justify giving them moral priority. They argue that humans should be given priority because they possess greater cognitive capacities, self-consciousness, moral agency, the ability to form deep emotional relationships with each other, and so forth (e.g., Kant, 1797/2017, 6:433; Quinn, 1984). But as critics often point out, some animals may also possess these capacities, at least in rudimentary form. And, more importantly, moral anthropocentrism is clearly taken to apply even to those humans who do not fully possess these features, whether due to young age or because they suffer from severe cognitive disability. 
But moral anthropocentrism is not just a disputed moral view but also a psychological phenomenon. It captures the way the vast majority of people think about the relations between humans and non-humans. While recent research has made important advances in understanding the psychological factors shaping attitudes to animals, the psychological sources of moral anthropocentrism remain unclear. The aim of the present study was to systematically investigate this issue.

An account of moral anthropocentrism as a psychological phenomenon has potentially wider significance. Like many other ethical debates, the debate between defenders and critics of moral anthropocentrism shows no sign of agreed resolution. Uncovering the psychological factors that drive moral anthropocentrism cannot, of course, resolve this ethical debate about the relative moral status of humans and other beings. But it could contribute to this debate in at least two ways. First, some of the factors uncovered may be ones that can be widely agreed to be morally relevant — or irrelevant. For instance, the case for moral anthropocentrism may be weakened if it turns out that it is in fact driven by factors viewed as morally irrelevant by philosophers. Second, the factors or principles that philosophers postulate as supporting moral anthropocentrism may be in tension with the factors that actually drive common intuitions and beliefs. Greater understanding of the psychological underpinning of moral anthropocentrism can thus make certain ethical views less (or more) plausible.

\section{Is Moral Anthropocentrism Based in Mental Capacity or in Speciesism?}

Debates in moral philosophy suggest two broad competing explanations for moral anthropocentrism:

Mental Capacity. One possibility is that people value humans more than animals because humans possess (or typically possess) morally valuable properties that animals lack 
but which other beings could potentially have. A salient example is the view that humans are morally more valuable than animals because they have advanced mental capacities which animals lack. These include complex language and thoughts, the ability to plan for the future, to make deliberate and autonomous judgments (including moral evaluations), and complex emotions. Capacities such as these are often regarded as being required to be considered a 'person' who is granted full moral status (e.g., Kant, 1797). On this hypothesis-which we call the mental capacity principle - people value humans more than animals because only humans have advanced mental capacities.

The mental capacity principle comes in two versions. The most straightforward version is what we call the individual mental capacity principle, which says that individual humans are viewed as morally more important than individual animals because humans have more advanced mental capacities (e.g., Kant, 1797). If this view were the primary source of moral anthropocentrism, we would expect people to regard non-human individuals that have mental capacities similar to those of humans, such as intelligent chimpanzees or extraterrestrials, to be as morally important as humans.

However, one problem for that hypothesis is that some humans are treated as possessing full moral status despite having less advanced mental capacities than many animals. Familiar examples are infants and severely cognitively impaired people—often referred to as 'marginal cases'. One possible reason why such people are granted full moral status is that they belong to a species whose members typically have advanced mental capacities (e.g., Cohen, 1986; Finnis, 1997; Scanlon, 1998). This more complex hypothesiswhich we call the species-typical mental capacity principle - says that people value humans more than animals because humans typically have more advanced mental capacities than animals. If that hypothesis were true, we would expect people to regard members of a species 
(e.g., an extraterrestrial species) whose typical members are as intelligent as humans to have a moral status on par with that of humans, regardless of their individual capacities.

Speciesism. A second possibility is that people value humans more than animals not because humans possess certain valuable properties that other animals lack but just because humans are members of the species Homo sapiens (cf., Singer, 1975, 1979). This tendency to assign moral status to beings on the basis of mere species-membership is referred to as speciesism (Caviola et al., 2019, 2020). But speciesism can take two different forms, and they have been so far rarely distinguished in discussions about speciesism (for an exception cf. Horta, 2010).

The first form of speciesism is what we call species-relativism: the view that all individuals should prioritize members of their own species over others because we have special duties to members of our own species (Pugh et al., 2013; Williams, 2009). While this general 'relativist' principle says that humans should prioritize humans over non-humans, it also says that members of other species should prioritize their fellow species-memberspotentially even at the expense of humans. Hence on this view, how someone should prioritize between different species depends on the species they belong to.

We call the second form of speciesism species-absolutism: treating humans are more valuable than non-humans simply because they are humans. Unlike species-relativism, According to species-absolutism even non-humans should be expected to prioritize humans over members of their own species, even when the humans have the same, or indeed weaker, mental capacities. In this sense, it is an absolute, rather than a relative, claim about why humans should be given priority. 


\section{Previous Research}

While moral philosophers have debated possible reasons for prioritizing humans over animals at great length, psychologists have only relatively recently begun to investigate people's moral attitudes towards animals (e.g., Amiot \& Bastian, 2015; Crimston et al., 2016; Dhont et al., 2014, 2019; Kasperbauer, 2017; Opotow, 1993; Plous, 1993). Previous research has proposed that social psychological intergroup theories that describe human-human forms of outgroup mistreatment (e.g., Bandura, 1999; Sidanius \& Pratto, 1999) also apply to human-animal relations. While previous research has demonstrated that people value humans more than animals (e.g., Caviola et al., 2019, 2020), there has not yet been any attempt to directly and systematically study the different factors that underlie moral anthropocentrism. However, several recent studies offer some support for both the mental capacity principle and for speciesism.

First, research has found that when animals are perceived to have lower mental capacities, such as lower intelligence and diminished capacity for suffering, they tend to be attributed lower moral status (Caviola et al., 2019, 2020; cf. Goodwin, 2015). In the context of meat consumption, it has been shown that denial of animals' capacity to suffer (a process referred to as de-mentalizing) is a key mechanism leading people to attribute lower moral status to food animals (Bastian et al., 2012; Bratanova et al., 2011; Loughnan et al., 2010). De-mentalizing appears to be a strategy that people unconsciously employ to reduce the cognitive dissonance they experience when confronted with the inconsistency of eating certain animals but loving other animals (the meat paradox; Piazza \& Loughnan, 2016). Thus, people's tendency to deny the capacity to suffer is stronger for animals classified as food than for pet animals (Bilewicz et al., 2011). In sum, this body of research suggests that people indeed value animals more the more advanced their perceived mental capacities are. 
However, these prior studies did not investigate whether this preference for more advanced mental capacities also explains why people value humans more than animals. Would people value non-humans with mental capacities equivalent to or even greater than that of humans?

Second, research has shown that people do exhibit speciesism. For example, people are more willing to help humans than animals even after statistically accounting for the differences in perceived mental capacity levels of humans and animals (Caviola et al., 2019). Furthermore, people are more willing to harm animals than to harm humans, even in cases where the humans are severely cognitively impaired or even lack any mental capacities (e.g., persistent vegetative state patients) (Caviola et al., 2020). This tendency is partly driven by speciesism - a trait which has the same psychological roots as other forms of prejudice against outgroups (Caviola et al., 2019; Dhont et al., 2016). However, while this line of research suggests that people at least to some extent value humans more than animals because of their species-membership, prior work has not distinguished the two forms of speciesism outlined above. Do people value humans more just because they are members of their own species (species-relativism) or because they believe humans are morally more important tout court (species-absolutism)?

\section{The Present Research}

Discussions of humans' sense of moral superiority over animals often assume that this attitude has a single source. The existing evidence, however, suggests that there might be multiple factors driving moral anthropocentrism. Our guiding hypothesis was therefore that both the mental capacity principle and speciesism play a role. Our aim was to clarify the extent to which each of these factors drives moral anthropocentrism while carefully 
distinguishing the different variants of these factors that we distinguished above-individual vs. species-typical mental capacities, and species-relativism vs. species-absolutism.

While the familiar contrast between humans and existing animals is the most dramatic real-life manifestation of moral anthropocentrism, philosophers have long recognized that we cannot fully understand moral anthropocentrism simply by investigating this contrast, since (nearly all) humans have numerous features that may potentially distinguish them from (nearly all) existing animals, meaning that it is hard to determine which ones primarily drive the phenomenon. The special case of humans with atypically low cognitive capacities only partly disentangles species-memberships from potential grounds for elevated moral status since, as mentioned above, even these humans still bear the distinction of belonging to a species whose typical cognitive capacities are superior (e.g., Cohen, 1986; Finnis, 1997; Scanlon, 1998). To further complicate things, since actual animals are not moral agents who can reason about right and wrong, real-life questions about how to treat humans compared to other beings always involve choices made by humans. A more systematic investigation of moral anthropocentrism therefore requires us to consider some (currently) hypothetical scenarios: (i) pitting humans against animals or other non-humans who have equal or even greater capacities; (ii) pitting humans against non-humans belonging to species that vary in their typical capacities; and (iii) pitting moral choices made by humans against such choices made by non-human agents.

In the debate in animal ethics, such scenarios may feature familiar animals with artificially enhanced cognitive capacities (McMahan, 2001) or extraterrestrials who possess equal or greater capacities (Williams, 2009). In the context of investigating the psychological basis of moral anthropocentrism, such hypothetical scenarios may seem distant from the experiences that shape people's moral views. But recent psychological work on moral 
decision-making often makes effective use of far-fetched scenarios to uncover the processes underlying moral judgment (e.g., far-fetched scenarios involving runaway trolleys, Greene, 2014), and similar scenarios involving hypothetical extraterrestrials have been used in psychological experiments before and have revealed findings in line with vignettes that relied on more realistic scenarios (e.g., Caviola et al., 2020; Sytsma \& Machery, 2012). For example, in a series of experiments, Starmans and Friedman (2016) investigated people's judgments about the ownership of humans and non-human entities, including aliens, robots and human-like biological creations. Furthermore, studies with children sometimes use anthropomorphized aliens as stimuli (Rottman \& Kelemen, 2012), which suggests that people are already capable of imagining such hypothetical beings at a young age. In the following studies, we begin by investigating the factors driving moral anthropocentrism using more realistic scenarios. On the basis of the results of these studies we then proceed to use scenarios that are less realistic in order to further disentangle factors that cannot be distinguished in currently realistic settings.

All our studies involve moral dilemmas where participants are asked to prioritize between two individuals that both require urgent help. When participants prioritize one individual over another, we will conclude that they see this individual as morally more important than the other. For simplicity reasons, we use the term 'value' for any form of higher moral status, whether that status reflects greater value in a cost-benefit analysis, associated rights that take precedence over others' rights or claims, or more demanding duties to aid that individual.

We use both familiar animals (chimpanzees and monkeys) and hypothetical beings in our scenarios. In Studies 1a-b we test the individual mental capacity principle by presenting participants with moral dilemmas involving humans and chimpanzees with different mental 
capacity levels (including chimpanzees with advanced mental capacities). In Study 2 we test the species-typical mental capacity principle, according to which moral status is determined by the typical mental capacity level of a species. Here we rely on moral dilemmas that contrast humans with members of hypothetical species (extraterrestrials) with either typically advanced or typically basic mental capacities. In Studies 3a-b we test the species-relativism and the pro-human species-absolutism hypotheses, by asking who humans and non-humans, respectively, should prioritize in identical moral dilemmas that involve human and nonhuman beneficiaries. In Study 4, we extend our question to contexts involving harm, and contrast attitudes to animals vs. to other non-humans.

Participant Recruitment. For all reported studies, we recruited participants from Amazon MechanicalTurk (MTurk). We collected the data through the platform Positly, which is a front-end platform that recruits MTurk participants. Positly includes additional proprietary quality metrics (https://www.positly.com/participants/). Concretely, Positly by default blocks duplicate and suspicious IP addresses, requires an approval rate of above $96 \%$ and at least 500 HITs, and requires participants to consistently pass attention checks.

Open Practices. Reports of all measures, manipulations, and exclusions, as well as all data, analysis code, and experimental materials are available for download at: https://osf.io/6ncb8/?view_only=874b1deb84884a3c8d035694057889ef. Studies 1a, 1b, 2, 3a and $3 \mathrm{~b}$ were pre-registered. In all studies, the data was analyzed only once data collection was completed, at which point no further data were collected.

Ethics Statement. For all studies, relevant ethical guidelines were followed, and the research was approved through the University of Oxford's Central University Research Ethics Committee. 


\section{Study 1a: Individual Mental Capacity}

In Study 1a, we aimed to test whether people value humans more than animals primarily because humans have higher mental capacities than animals. If humans are judged to be morally more valuable than animals because they possess higher relative mental capacities, then individual animals whose mental capacities are at the same level as individual humans should be judged to be as morally valuable as those humans. Moral philosophers have long argued that people apply the mental capacity principles inconsistently because they prioritize mentally impaired humans over animals with comparable mental capacities. However, it is possible that many people reject this assumption of equivalent mental capacities, or that they assume that mentally impaired humans have a greater capacity for suffering. In study 1a, we set out to investigate such prioritization questions more rigorously by devising contexts where certain non-humans (chimpanzees) are directly pitted against mentally impaired humans while manipulating mental capacity levels in a broadly realistic range. We hypothesized that people would continue to value humans more than animals even in cases where the animals in question were stipulated, in this way, to have similar mental capacities to the humans in question (cf. Caviola et al., 2019; 2020). This study was preregistered at https://aspredicted.org/VZY JXY.

\section{Methods}

Participants. We recruited 296 US American participants online via MTurk. They received $\$ 0.75$ for their participation. Forty-six were excluded for failing one of two attention checks or our comprehension checks described below, leaving a final sample of 250 people $\left(140\right.$ female; $\left.M_{\text {age }}=38.5, S D_{\text {age }}=11.89\right)$. We aimed to recruit 300 participants. This number accounted for the fact that a sizable fraction would be excluded. 
Materials and Procedure. This study employed a within-subjects design.

Participants first read a short text that highlighted both actual scientific research showing that some chimpanzees have far greater mental capacities than previously assumed, and the possibility that in the future chimpanzees could be trained to exhibit even more impressive mental capacities. Participants were then presented with a scenario in which scientists had brought together mentally impaired humans and chimpanzees to study how they solve puzzles. The humans and the chimpanzees were said to either have 'lower mental capacity' or 'higher mental capacity'.

Lower mental capacity was described as follows: "Humans and chimps with lower mental capacity have fairly limited mental capacities. They have only a rudimentary way of communicating and of forming social relationships. They are not self-aware, cannot judge morally right from wrong, and cannot form advanced thoughts or plan for the future. Instead, they primarily respond to the environment based on impulse and instinct. Finally, these humans and chimps cannot experience complex feelings such as grief and guilt.”

Higher mental capacity was described as follows: "By contrast, humans and chimps with higher mental capacity both have more advanced mental capacities than humans and chimps with lower capacities. They have some language skills and can form relatively complex social relationships. They are somewhat self-aware, have a basic sense of the difference between right and wrong, and can even make autonomous decisions and basic plans for the future. Finally, these chimps and humans can experience complex feelings such as grief and guilt."

It was emphasized that "a human with lower mental capacity and a chimp with lower mental capacity have the exact same mental capacities" and that "a human with higher mental capacity and a chimp with higher mental capacity have the exact same mental capacities". 
Participants were also informed that all individuals, regardless of whether they are human or chimpanzee and regardless of their mental capacity level, are equally capable of experiencing pain and suffering.

On the next page, participants were asked to rate the mental capacities of all four types of beings on a slider each (0-100). Next, they were asked to rate the capability of experiencing pain of all four types of beings on a slider each (0-100). These measures were used as comprehension checks. As pre-registered, we excluded participants (41 in total) from the analyses who rated the mental capacities (but not the suffering capability) of chimpanzees 5 or more point lower than humans with the same mental capacity level. Note that downgrading the mental capacities of animals despite being informed about their intelligence levels, as well attributing to animals a reduced capacity to suffer (e.g., Bastian et al., 2012), are phenomena that can be taken in themselves as instances of motivated reasoning for the purpose of animal devaluation. However, in our specific study we wanted to exclude such processes to investigate ascription of moral value even if mental capacities are perceived as equal between humans and animals. In other words, we wanted to investigate the pure effect of mental capacities, in absence of motivated reasoning. Note that in the Supplementary Materials we reported two additional versions of the same analyses, one in which we also excluded participants who rated the suffering capacity of chimpanzees 5 or more point lower than humans and one without any exclusions. The pattern of results remains the same in both cases.

Next, participants were presented with six dilemmas in randomized order. In each dilemma, two individuals were said to be infected with a non-contagious lethal disease. Participants had to decide which of the two individuals they would rather give life-saving medicine to, if they could only one save one of them. Two of the dilemmas were intra-species 
cases: a human with lower mental capacity vs. a human with higher mental capacity; a chimpanzee with lower mental capacity vs. a chimpanzee with higher mental capacity. Four of the dilemmas were inter-species cases: a human with lower mental capacity vs. a chimpanzee with lower mental capacity; a human with higher mental capacity vs. a chimpanzee with higher mental capacity; a human with lower mental capacity vs. a chimpanzee with higher mental capacity; a human with higher mental capacity vs. a chimpanzee with lower mental capacity. Participants were asked: "From an ethical perspective, if you can save only one of these two individuals, which one should you save?“, and responded on a 7-point scale ( 1 = Definitely [individual A], $4=$ It is equally ethically right to save the one or the other, $7=$ Definitely [individual $B]$ ).

Finally, participants responded to the Speciesism Scale (see Supplementary Materials for results) and demographic questions. We report all measures, manipulations, and exclusions.

Throughout all our studies, we use two methods to test whether participants have a tendency to prioritize one individual over another. First, we look at the proportion of participants who prioritize the first individual (response below the mid-point) compared to the proportion of participants who did not, either because they believe saving either is equally right (response on the mid-point) or because they prioritized the second individual (response above the mid-point). Where helpful we conduct Chi-square tests to check for significant differences in proportions. Second, we look at the mean responses. Where helpful, we conduct one-sample t-tests against the mid-point to check if the mean responses are significantly above or below the mid-point. In many cases, the conclusions of both analyses converge. In the cases where they don't, we discuss possible interpretations. 


\section{Results}

In all four inter-species cases (Figure 1a, Table 1), the majority of participants prioritized humans over chimpanzees regardless of their relative mental capacities. For example, $78.0 \%$ of participants prioritized a human with higher mental capacity over a chimpanzee with higher mental capacity, whereas the remaining participants did not (either indicating that both options are equally right, or prioritizing the chimpanzee), $\chi^{2}(1)=78.4, p$ $<.001$. Similarly, $66.0 \%$ prioritized a human with lower mental capacity over a chimpanzee with higher mental capacity, $\chi^{2}(1)=25.6, p<.001$. In this dilemma, only $13.6 \%$ prioritized the mentally advanced chimpanzee over the mentally weaker human. It is noteworthy that in cases where the human and chimpanzee had equal mental capacity levels, roughly a fifth of participants indicated that either option was ethically right (Table 1).

Next, we conducted parametric analyses with the mean responses (Table 1). The average participant prioritized humans over chimpanzees regardless of their relative mental capacities $(p<.001$ in all cases). However, this tendency was stronger in cases where the human had higher mental capacities than the chimpanzee. For example, participants' tendency to prioritize a human with higher mental capacities over a chimpanzee with lower mental capacities was more pronounced than their tendency to prioritize a human with higher mental capacities over a chimpanzee with similarly higher mental capacities, $t(249)=5.12, p$ $<.001, d=.33$.

In the two intra-species cases (Figure 1b, Table 1), the majority of participants indicated that saving either of the two individuals was ethically right. However, a substantial proportion prioritized the individual with the higher mental capacities over the individual with the lower mental capacities of the same species. This tendency was more pronounced in 
the dilemma that pitted two chimpanzees against each other than in the dilemma that pitted two humans against each other, $\chi^{2}(1)=12.01, p<.001$.

Next, we conducted parametric analyses with the mean responses. The average participant prioritized the chimpanzee with higher mental capacities over the chimpanzee with lower mental capacities, $t(249)=-13.01, p<.001, d=.82$, and the human with higher mental capacities over the human with lower mental capacities, $t(249)=-9.98, p<.001, d=$ .63. Participants' tendency to prioritize individuals with higher mental capacities over individuals with lower mental capacities of the same species was stronger for chimpanzees than humans, $t(249)=4.36, p<.001, d=.27$.

\begin{tabular}{llllll}
\hline A & B & Prioritize A & Equal & Prioritize B & M (SD) \\
\hline Human-lower & Chimpanzee-lower & $77.2 \%$ & $20.4 \%$ & $2.4 \%$ & $2.08(1.42)$ \\
Human-lower & Chimpanzee-higher & $66.0 \%$ & $20.4 \%$ & $13.6 \%$ & $2.65(1.82)$ \\
Human-higher & Chimpanzee-lower & $85.6 \%$ & $13.6 \%$ & $0.8 \%$ & $1.72(1.19)$ \\
Human-higher & Chimpanzee-higher & $78.0 \%$ & $18.8 \%$ & $3.2 \%$ & $2.05(1.43)$ \\
Human-higher & Human-lower & $32.4 \%$ & $67.2 \%$ & $0.4 \%$ & $3.24(1.21)$ \\
Chimpanzee-higher & Chimpanzee-lower & $48.0 \%$ & $51.2 \%$ & $0.8 \%$ & $2.89(1.35)$ \\
\hline
\end{tabular}

Table 1. Proportions of participants who either prioritized individual A, who indicated that it is equally ethical to save either, or who prioritized individual B. "higher" and "lower" indicate the mental capacity level of the respective individuals. The last column contains the means and standard deviations. (Study 1a) 
Human (1) vs. Chimpanzee (7)

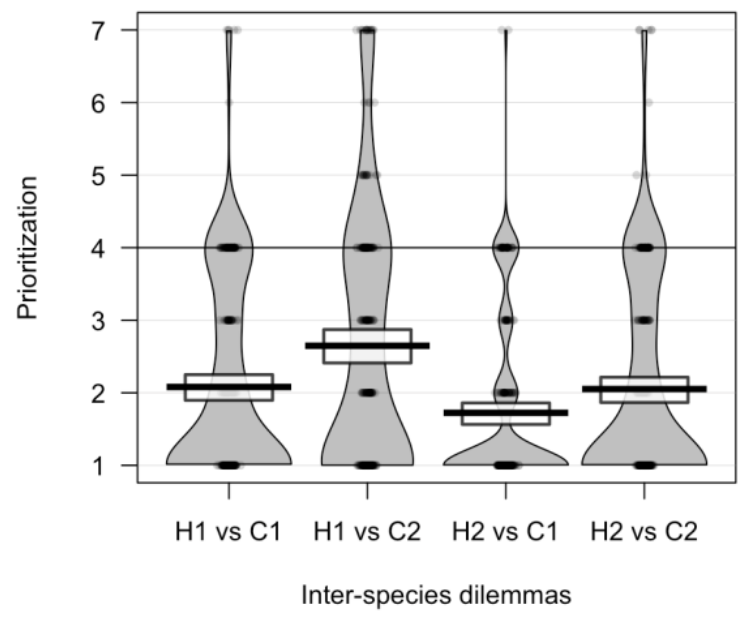

Figure 1a. Most participants prioritized humans over chimpanzees regardless of their relative mental capacity levels. This tendency was stronger the in cases where the humans had higher mental capacities than the chimpanzees. H1 stands for Human with lower mental capacities, C2 stands for chimpanzee with higher mental capacities, and so on. (Study 1a)
Higher (1) vs. Lower (7)

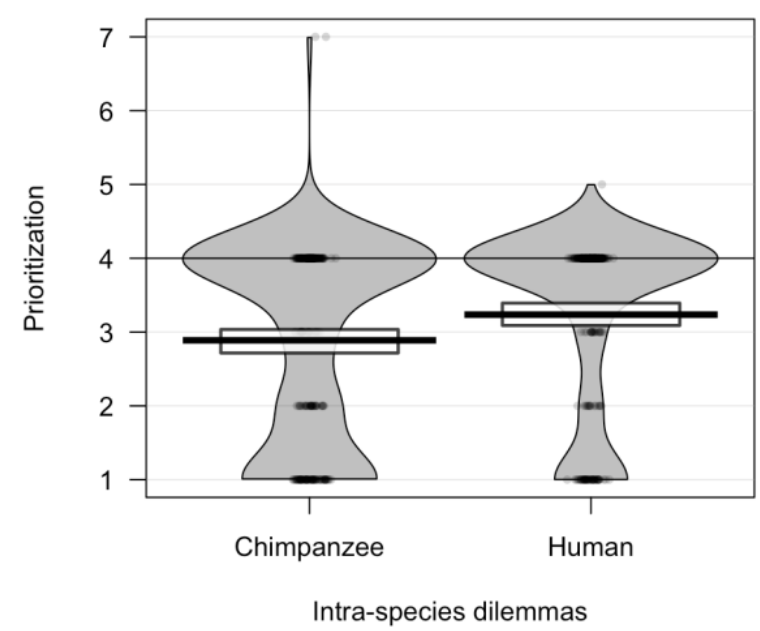

Figure 1b. Participants were divided on whether to prioritize individuals with higher mental capacities over individuals wither lower mental capacities of the same species. Many believed it was equally right to save either. On average, participants tended to prioritize higher mental capacities more in chimpanzees than in humans. (Study 1a)

\section{Discussion}

The results of Study 1a suggest that it is not the case that people value humans more than animals primarily because individual humans have higher mental capacities than individual animals. In all cases where humans were pitted against chimpanzees, participants on average prioritized helping the human over the chimpanzee. This was even true when the 
chimpanzee had a higher level of mental capacity than the human. However, it became also clear that participants still gave some moral weight to higher levels of mental capacities. Specifically, we found that they tended to prioritize humans with higher mental capacity levels over chimpanzees with lower mental capacity levels to a greater extent than they prioritized humans over chimpanzees with the same mental capacity levels. Thus, this study suggests that, while people grant higher moral status to individuals with higher mental capacity levels, species-membership trumps the effect of having higher or lower relative mental capacity levels.

Furthermore, we also found that people, on average, had a stronger tendency to prioritize a chimpanzee with higher mental capacities over a chimpanzee with lower mental capacities than to prioritize a human with higher mental capacities over a human with lower mental capacities. This suggests that people have a stronger tendency to apply the mental capacity principle to animals than to humans - a tendency that could itself be seen as speciesist. In line with that, we found that scores on the Speciesism Scale (Caviola et al, 2019) predicted the tendency to prioritize a chimpanzee with higher mental capacities over a chimpanzee with lower mental capacities. However, Speciesism Scale scores did not correlate with the tendency to prioritize a human with higher mental capacities over a human with lower mental capacities. Unsurprisingly, scores on the Speciesism Scale predicted the tendency to prioritize the human over the chimpanzee in all inter-species cases (see Supplementary Materials for more details).

We wish to clarify that we do not, in fact, hold that the mental capacities of cognitively impaired humans are in fact exactly the same as those of any non-human. This somewhat artificial stipulation was introduced for methodological reasons: more precise language that spoke instead of capacities that are 'broadly equivalent' or 'identical on 
average' could suggest to participants that there might be further, more specific mental features that might morally distinguish the humans and non-humans in these scenarios. Our simplifying stipulation aimed to remove (or at least reduce) the possibility that such considerations would influence people's responses. Future research could more directly investigate the possibility that even when people perceive non-humans as having overall the same mental capacity levels as some humans, they nevertheless think that even cognitively impaired humans enjoy certain finer-grained cognitive capacities that endow them with higher moral status (see, for example, Jaworska \& Tannenbaum, 2014). More generally, we recognize that hypothetical scenarios that identify (or more generally compare) the capacities of cognitively impaired humans and of (certain) non-humans, and that involve tragic prioritization choices, are ethically sensitive and we have tried to approach them with great care. Some of our scenarios involve specific non-humans that are presented as having cognitive capacities that are as high as those of some humans; but they do not downplay the capacities of any category of humans. We believe, moreover, that studies such as ours help shed light not just on the factors that shape people's views on the moral status of non-human animals, but also on how people perceive the moral status of cognitively impaired individuals.

A potential limitation of this study, as well as the other studies presented in this paper, is that participants may have assumed that the humans and chimpanzees differ on some specific mental capacity while still being equal in their average mental capacities. For example, participants may have believed that humans tend to have better moral judgment abilities but that chimpanzees have better practical reasoning abilities, such that on average they have the same mental capacities. And to the extent that people place more moral weight on certain specific mental capacities (e.g., moral judgment abilities) than on others, this could 
in principle explain our results. However, we consider this a relatively unlikely explanation since participants were explicitly told that a human with lower (or higher) mental capacity and a chimp with lower (or higher) mental capacity have the exact same mental capacities, and since our explanatory text described a range of common cognitive, affective and moral capacities. Moreover, the scenarios involved humans with impaired mental capacities - with the lower capacity group described as having severely impaired capacities—which should greatly limits to space for participants to nevertheless imagine that these humans nevertheless possess further distinctive capacities.

However, this advantage of the study is also a limitation since we only looked at mental capacity levels below the advanced mental capacity levels of typical humans. In particular, it is possible that people would value animals as much as humans in less realistic cases where individual animals have mental capacities that are similar to those of typical humans. In Study 1b we look at such cases.

\section{Study 1b: Individual Mental Capacity on Advanced Levels}

As just mentioned, people might value humans more than animals primarily because humans have advanced mental capacities that allow for complex thoughts and emotions, deliberate planning, and autonomous choice, whereas animals do not, in line with the Kantinspired view that only autonomous moral agents ('persons') have 'full' moral status. If humans are judged to be morally more valuable than animals because they possess such advanced mental capacities, then hypothetical animals whose mental capacities are at the human level should be judged to be as morally valuable as humans. Another implication of the individual mental capacity principle is that animals with above human-level mental capacities might be viewed as even morally more valuable than humans (such an ethical view 
is explored by Douglas, 2013). In Study 1b, we test for these possibilities. Our hypothesis was again that people would continue to value humans more than animals even if the animal has human-level or above-human-level mental capacities. Together, these two predictions meant that we did not think that the individual mental capacity principle could fully explain moral anthropocentrism. To test these hypotheses, we need to look at hypothetical cases involving currently unrealistically mentally advanced animals. Apart from this necessity of an unrealistic scenario, the setup of Study $1 \mathrm{~b}$ was similar to Study 1a. This study was preregistered at https://aspredicted.org/SVH_37R.

\section{Methods}

Participants. We recruited 308 US American participants online via MTurk. They received $\$ 0.85$ for their participation. Fifty-five were excluded for failing one of two attention checks or one of our comprehension checks described below, leaving a final sample of 253 people $\left(140\right.$ female; $\left.M_{\text {age }}=38.5, S D_{\text {age }}=11.89\right)$. We aimed to recruit 300 participants.

Materials and Procedure. The study had a similar within-subject design as Study 1a. But instead of six dilemmas, all participants were presented with fifteen different dilemmas in randomized order. All dilemmas featured two individuals that were pitted against each other. Participants were told that both individuals suffer from a potentially fatal disease and that they had to decide which one they would prioritize to save if only one life could be saved.

The two individuals were either a human and a chimpanzee (nine 'inter-species' dilemmas), two humans (three 'intra-species' dilemmas) or two chimpanzees (three 'intraspecies' dilemmas). The individuals either had low (animal-level), high (human-level), or extraordinary (above-human-level) mental capacity levels. Consequently, some dilemmas involved cases such as chimpanzees with human-level or even above-human-level mental capacities. While such scenarios are obviously unrealistic, we attempted to place them within 
a moral realistic context by first presenting participants with a short text that described actual research aiming to radically enhance the cognitive capacities of humans, and which discussed the possibility that such technologies could also be used to enhance some chimpanzees, and the ethical challenges this could raise.

In each dilemma, participants had to indicate which individual they would prioritize saving (by providing a medicine) on a 7-point scale $(1=$ Definitely $A, 4=$ It is equally ethically right to save the one or the other, $7=$ Definitely $B$ ), where A and B refer to the two individuals respectively and differ in each dilemma.

Finally participants responded to the Speciesism Scale (see Supplementary Materials for results) and demographic questions. We report all measures, manipulations, and exclusions.

\section{Results}

We first looked at the nine 'inter-species' dilemmas in which humans were pitted against chimpanzees (Table 2; Figure 2a). In total there were nine such dilemmas, of three different types. We created averaged scores for the three types and used them for all further analyses. These were: three 'higher-humans' dilemmas, where the humans had higher capacities than the chimpanzees (extraordinary human vs. high chimpanzee, extraordinary human vs. low chimpanzee, high human vs. low chimpanzee; $\alpha=0.92$ ), three 'higherchimpanzees' dilemmas, where the chimpanzees had higher mental capacities (extraordinary chimpanzee vs. high human, extraordinary chimpanzee vs. low human, high chimpanzee vs. low human; $\alpha=0.90$ ), and three 'equal-capacities' dilemmas, where the humans and chimpanzees had equal mental capacities (extraordinary human vs. extraordinary chimpanzee, high human vs. high chimpanzee, low human vs low chimpanzee; $\alpha=0.94$ ). 
In line with our hypothesis, we found that the majority of participants prioritized humans over chimpanzees regardless of their relative mental capacities. The following analyses were conducted with a binary variable. $85.0 \%$ of participants prioritized humans over chimpanzees in the higher-humans dilemmas, whereas the remaining participants did not (either indicating that both options are equally right, or prioritizing the chimpanzee), $\chi^{2}(1)=$ $123.83, p<.001$. Similarly, $70.0 \%$ of participants prioritized humans over chimpanzees in the equal-capacities dilemmas. $\chi^{2}(1)=40.32, p<.001$. It is noteworthy that in the equalcapacities dilemmas, roughly a quarter of participants indicated that either option was ethically right. Finally, $53.3 \%$ of participants prioritized humans over chimpanzees in the higher-chimpanzees dilemmas, $\chi^{2}(1)=1.14, p=.29$. Note that even though only slightly more than half of participants chose to prioritize humans with lower mental capacities over chimpanzees with higher mental capacities, less than a third chose to prioritize chimpanzees with higher mental capacities over humans with lower mental capacities (Table 2).

An analysis of the mean responses yielded similar results. The average participant tended to prioritize humans over chimpanzees in the higher-human dilemmas, $t(252)=-$ 26.02, $p<.001, d=1.64$, in the equal-capacities dilemmas, $t(252)=-17.0, p<.001, d=1.07$, as well as in the higher-chimpanzees dilemmas, $t(252)=-6.64, p<.001, d=.42$. A one-way ANOVA revealed significant differences across these three averaged scores, $F(2,504)=$ $124.49, p<.001$. Follow-up test revealed that all three scores significantly differ from each other $(p<.001$ in all cases $)$.

Next, we looked at the six 'intra-species' dilemmas where individuals of the same species, but with different mental capacities, were pitted against each other (extraordinary vs. high, extraordinary vs. low, high vs. low; Table 2; Figure 2b). Again, we created two 
averaged scores composed of the three dilemmas involving humans $(\alpha=0.87)$, and the three dilemmas involving chimpanzees $(\alpha=0.88)$.

The following analyses were conducted with a binary variable. In the human-vshuman dilemmas, roughly half of participants (51.4\%) prioritized the human with higher mental capacities over the human with lower mental capacities or did not, $\chi^{2}(1)=0.19, p=$ .66. It is noteworthy that $47.4 \%$ of participants believed that it is equally right to save either the human with higher mental capacities or the human with lower mental capacities. By contrast, in the chimpanzee-vs-chimpanzee dilemmas, significantly more than half of participants $(61.7 \%)$ prioritized the chimpanzee with the higher mental capacities over the chimpanzee with lower mental capacities, $\chi^{2}(1)=13.76, p<.001 .37 .1 \%$ of participants believed that it is equally right to save either the chimpanzee with higher mental capacities or the chimpanzee with lower mental capacities. This difference between the two types of dilemmas was significant, $\chi^{2}(1)=5.026, p=.03$.

An analysis of the mean responses yielded similar results. The average participant prioritized the human with higher mental capacities over the human with lower mental capacities, $t(252)=-11.76, p<.001, d=.74$, and the chimpanzee with higher mental capacities over the chimpanzee with lower mental capacities, $t(252)=-15.8, p<.001, d=$ 99. However, their tendency to prioritize the human with higher mental capacities over the human with lower mental capacities was significantly weaker than their tendency to prioritize the chimpanzee with higher mental capacities over the chimpanzee with lower mental capacities, $t(252)=6.42, p<.001, d=.31$.

\begin{tabular}{lllll}
\hline Dilemma type & Prioritize A & Equal & Prioritize B & M (SD) \\
\hline
\end{tabular}




\begin{tabular}{lcccc}
\hline Higher human & $85.0 \%$ & $13.4 \%$ & $1.6 \%$ & $2.01(1.22)$ \\
Equal capacities & $70.0 \%$ & $26.9 \%$ & $3.1 \%$ & $2.50(1.41)$ \\
Higher chimpanzee & $53.3 \%$ & $17.8 \%$ & $28.9 \%$ & $3.26(1.77)$ \\
Intra-Humans & $51.4 \%$ & $47.4 \%$ & $1.2 \%$ & $3.16(1.14)$ \\
Intra-Chimpanzees & $61.7 \%$ & $37.1 \%$ & $1.2 \%$ & $2.80(1.21)$
\end{tabular}

Table 2. Proportions of participants who either prioritized individual A, who indicated that it is equally ethical to save either, or who prioritized individual B. In the upper three dilemma types, individual A is a human and individual B a chimpanzee. In the lower two dilemma types, individual $\mathrm{A}$ is the one with the higher mental capacities and individual $\mathrm{B}$ the one with the lower mental capacities. The last column contains the means and standard deviations. (Study 1b)

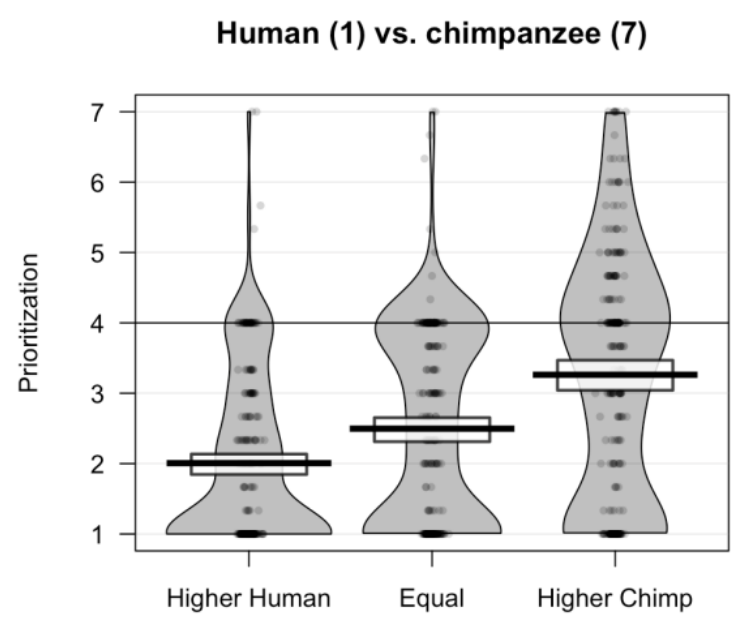

Inter-species dilemmas
Higher (1) vs. lower (7) mental capacities

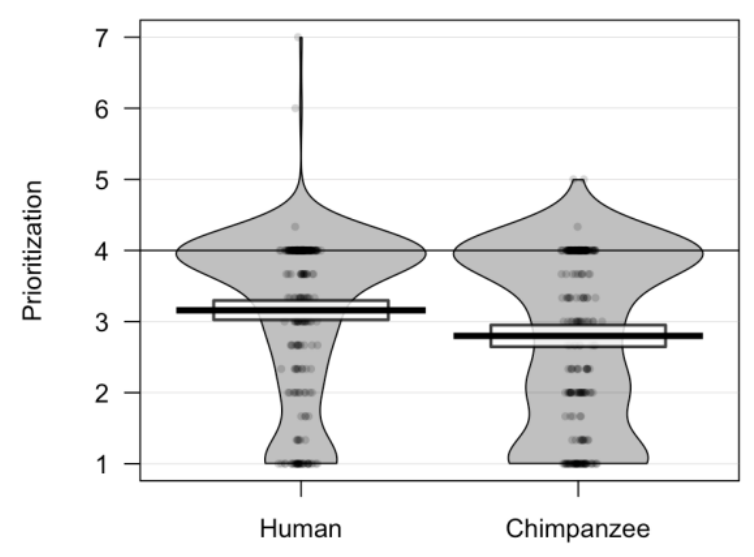

Intra-species dilemmas 
Figure 2a. Most participants prioritized

humans over chimpanzees in all types of

dilemmas: in those where the humans had stronger mental capacities, in those where humans and chimpanzees had equal mental capacities, and in those where chimpanzees had stronger mental capacities. (Study 1b)
Figure $2 b$. Participants were divided on whether to prioritize individuals with higher mental capacities over individuals wither lower mental capacities of the same species. Many believed it was equally right to save either. On average, participants tended to prioritize higher mental capacities more in chimpanzees than in humans. (Study 1b)

\section{Discussion}

In line with Study 1a, Study 1b further supports our hypothesis that most people prioritize humans over animals regardless of their relative mental capacity levels. We found that most participants prioritized humans over chimpanzees even in extreme cases where the chimpanzee's mental capacity level was on par or even higher than typical humans. Thus, our results suggest that it is not the case that people value humans more than animals primarily because individual humans have more advanced mental capacities compared to animals.

However, as in Study 1a, we found that participants did take mental capacity levels into account. Their tendency to prioritize humans over chimpanzees was weaker the higher the relative mental capacity level of the chimpanzees was. In the dilemmas in which the chimpanzee had higher mental capacities than the human only about half $(53.3 \%)$ of participants prioritized the chimpanzee, whereas $17.8 \%$ believed it was equally right to save either and $28.9 \%$ even prioritized the chimpanzee. And again, in line with Study 1a, we found that the tendency to prioritize individuals with higher mental capacities over individuals with 
lower mental capacities of the same species was stronger when the individuals in question were chimpanzees than when they were humans.

To conclude, Studies 1a-b suggest that, while people grant higher moral status to individuals with higher mental capacities, species-membership trumps the effect of having higher or lower mental capacities. This conclusion is incompatible with the notion that people prioritize humans over animals simply because individual humans have higher mental capacities than animals. This means that there must be another reason for why people value humans more than animals.

\section{Study 2: Species-Typical Mental Capacity Level}

In Studies 1a-b we found that differences in individual mental capacity cannot be the main reason why people value animals less than humans. An alternative explanation is that people's valuation of an individual is based on the mental capacities of the typical member of the individual's species (rather than on the individual's own mental capacities). This speciestypical mental capacity principle has been defended by several prominent philosophers (see e.g., Cohen, 1986; Finnis, 1997; Kagan, 2019, ch. 5, Chapter 5; Scanlon, 1998). Since humans typically have advanced mental capacities and animals typically not, the speciestypical mental capacity principle is a plausible explanation for why people value all humans, even those with just basic mental capacities, more than animals. To our knowledge, this hypothesis has not been empirically tested before.

The hypothesis says that animals (e.g., chimpanzees or monkeys) with advanced or extremely advanced mental capacities are valued less than the typical human because they belong to a species whose typical mental capacities are weaker than those of the typical human. Similarly, it says that humans whose mental capacities are atypically basic for 
humans are still valued as much as other humans, who have the species-typical level of mental capacity. Hence, this hypothesis says that such humans, too, are valued more than animals with advanced or extremely advanced mental capacities.

Study 2 was designed to test this hypothesis. Our research question was: is people's valuation of individual members of a species based on the mental capacities of the typical member of that species? Our hypothesis — which was pre-registered at OSF was that participants would always prioritize humans over non-humans irrespective of the typical mental capacity level of the non-human species. In order to test this hypothesis, we asked participants to imagine hypothetical extraterrestrial species called Atlans (inspired by Sytsma and Machery, 2012), which were either described as typically having advanced mental capacities or, as typically having basic mental capacities. We introduced these hypothetical extraterrestrial species because we thought that participants would find it more credible that the typical member of a yet unknown extraterrestrial species has advanced mental capacities, than that the typical member of an animal species has advanced mental capacities. We hypothesized that in dilemmas when such beings are pitted against humans or each other, typical species-level would not make a difference: extraterrestrials with typically advanced and typically basic mental capacities would be treated similarly. We also included a third condition that contrasted monkeys to humans in order to investigate whether there would be differences in people's judgments between the conditions involving extraterrestrials and monkeys. This means we need to look again at unrealistic hypothetical cases involving currently unrealistically individuals. While we consider this the currently best way to test our specific hypothesis, it is important to keep in mind that some participants may find it hard to imagine such scenarios. 


\section{Method}

Participants. We recruited 614 US American participants online via MTurk. They received $\$ 0.72$ in payment for their participation. Fifty-five were excluded for failing a comprehension check, leaving a final sample of 559 people $\left(278\right.$ female; $M_{\text {age }}=39.2, S D_{\text {age }}=$ 11.96). Sample size was determined before any data analysis. We aimed to recruit 600 participants.

Materials and Procedure. We varied the type of non-human species that was pitted against a human (between-subjects) as well as the individual mental capacity levels of all beings (within-subjects) across six dilemmas: basic human vs. basic non-human, advanced human vs. advanced non-human, advanced human vs. basic non-human, basic human vs. advanced non-human, basic human vs. advanced human, basic non-human vs. advanced nonhuman. The non-human beneficiaries were either typically mentally advanced extraterrestrials, typically mentally basic extraterrestrials, or monkeys (which typically are mentally basic), depending on the between-subjects condition.

The instructions first described the typical mental capacity level of the species, e.g., "Like humans, typical Atlans can form advanced thoughts, have complex language, and can engage in cultural activities and politics (...)". Next, it was stated that there are a few special members of this species with more basic [advanced] mental capacities, e.g., "However, while most typical Atlans have comparable mental capacities to humans, a few special Atlans you will encounter, who are severely mentally disabled, have basic mental capacities. Their mental capacities are much more basic than those of a typical Atlan or of a typical human and are comparable to those of a typical monkey. (...)".

Therefore, the beneficiaries were either typical or atypical representatives of the respective species they belong to. This was crucial to test whether individuals with equal 
individual mental capacity levels were treated differently depending on their species' typical mental capacity level. Depending on the condition a mentally basic extraterrestrial was either a typical representative of its species (in the typically mentally basic extraterrestrial condition) or an atypical representative of its species (in the typically mentally advanced extraterrestrial condition).

Like in Studies 1a-b, for each dilemma participants were asked to indicate which of the two individuals they would prioritize helping if both required urgent help, but they could only help one. While participants in Studies 1a-b were told that they could save the life of one individual by providing a medicine, we used a more general phrasing in this and the following studies, with the aim of reducing potential confounders: "Sometimes we can only save the life, treat the illness, or relieve the pain of some but not of others. We then have to choose: whose life do we save, whose illness do we treat, and whose pain do we relieve?"(...) Suppose you were in a situation where you could only help one of these two individuals: $(A)$ A human that has basic mental capacities; (B) An Atlan that has basic mental capacities". Participants had to indicate on a 7-point scale which individual they should prioritize from an ethical standpoint $(1=$ Definitely A, $4=$ Flip a coin to decide, $7=$ Definitely $B)$.

\section{Results}

For each of the six dilemmas, we conducted a one-way ANOVA to test whether there were significant differences across the three conditions (Table 3, Figure 3). We found that for all dilemmas there were significant differences across the conditions exceptunsurprisingly — for the dilemma where a human was pitted against another human in all three conditions (thus, this dilemma was identical across the three conditions). Tukey HSD post-hoc analyses showed that none of the responses between the two extraterrestrial 
conditions differed significantly from each other (Table 3). This means that, in line with our main hypothesis, it made no difference whether the extraterrestrials belonged to a typically mentally advanced or typically mentally basic extraterrestrial species. This was true both regarding dilemmas where an extraterrestrial was pitted against a human and regarding dilemmas where two extraterrestrials were pitted against each other. However, the responses in the conditions where monkeys were pitted against humans differed significantly from the responses in the two conditions where extraterrestrials were pitted against humans (Table 3). The tendency to prioritize humans over non-humans was stronger when the non-human was a monkey than when it was an extraterrestrial.

For each condition (the monkey condition, the typically mentally advanced extraterrestrial condition, and the typically mentally basic extraterrestrial condition), we created averaged scores for the four dilemmas that pitted humans against non-humans. In the typically mentally advanced extraterrestrial condition, $55.1 \%$ prioritized the humans, $36.4 \%$ flipped a coin, and $8.6 \%$ prioritized the extraterrestrials. In this condition, the proportion who prioritized the humans was not statistically greater than the proportion who did not (either flipped a coin or prioritized the extraterrestrials), $\chi^{2}(1)=1.93, p=.16$. In the typically mentally basic extraterrestrial condition, $60.2 \%$ prioritized the humans, $32.0 \%$ flipped a coin, and $7.7 \%$ prioritized the extraterrestrials. In this condition, the proportion who prioritized the humans was statistically greater than the proportion who did not, $\chi^{2}(1)=7.56, p=.005$. Despite this, there was no direct statistically significant difference in the proportion of choices between these two conditions, $\chi^{2}(2)=1.00, p=.61$.

In the monkey condition, $85.3 \%$ prioritized the humans, $8.4 \%$ flipped a coin, and $6.3 \%$ prioritized the monkeys. In this condition, the proportion who prioritized the humans was statistically greater than the proportion who did not, $\chi^{2}(1)=95.42, p<.001$. The 
proportions of choices across the typically mentally basic extraterrestrial condition and the monkey condition were statistically significantly different, $\chi^{2}(2)=34.47, p<.001$. In particular, there were many more participants who prioritized the humans over the monkeys than there were participants who prioritized the humans over the typically basic extraterrestrials.

Next, we conducted analyses with the mean responses. The average participant prioritized humans over monkeys, $t(190)=-20.90, p<.001, d=1.51$, humans over typically mentally basic extraterrestrials, $t(186)=-10.75, p<.001, d=.80$, and even humans over typically mentally advanced extraterrestrials, $t(186)=-8.85, p<.001, d=.65$. The only exception was the case where the individual extraterrestrial had advanced and the human basic mental capacities, in which case the average participant flipped a coin, $t(186)=0.18, p$ $=.86, d=.01$.

We conducted three paired-sample $t$-tests to test whether the average participant was more likely to prioritize mentally advanced non-humans over mentally basic non-humans than they were to prioritize mentally advanced humans over mentally basic humans (as they were in Study 1a-b). That was indeed the case in all three conditions: the typically mentally advanced extraterrestrial condition, $t(186)=3.40, p<.001, d=.25$; the typically mentally basic extraterrestrial condition, $t(180)=4.71, p<.001, d=.35$; the monkey condition, $t(190)$ $=6.67, p<.001, d=.48$. 


\begin{tabular}{|c|c|c|c|c|c|c|c|c|c|c|c|}
\hline & \multicolumn{3}{|c|}{ Non-human species } & \multicolumn{2}{|c|}{$\begin{array}{l}\text { All three } \\
\text { conditions }\end{array}$} & \multicolumn{2}{|c|}{$\begin{array}{c}\text { Typically advanced } \\
\text { Atlans vs. typically } \\
\text { basic Atlans }\end{array}$} & \multicolumn{2}{|c|}{$\begin{array}{c}\text { Typically } \\
\text { advanced Atlans } \\
\text { vs. monkeys }\end{array}$} & \multicolumn{2}{|c|}{$\begin{array}{c}\text { Typically basic } \\
\text { Atlans vs. monkeys }\end{array}$} \\
\hline & $\begin{array}{l}\text { Typically } \\
\text { advanced } \\
\text { Atlans }\end{array}$ & $\begin{array}{l}\text { Typically } \\
\text { basic } \\
\text { Atlans }\end{array}$ & Monkeys & $F$ & $\eta_{p}^{2}$ & $b$ & $d$ & $b$ & $d$ & $b$ & $d$ \\
\hline $\begin{array}{l}\text { Basic human vs. } \\
\text { basic non- } \\
\text { human }\end{array}$ & $\begin{array}{l}3.02 \\
(1.39)\end{array}$ & $\begin{array}{c}2.77 \\
(1.50)\end{array}$ & $\begin{array}{c}1.86 \\
(1.40)\end{array}$ & $33.99^{* * *}$ & .11 & -0.25 & -.17 & $-1.15^{* * *}$ & -.82 & $-.90^{* * *}$ & -.62 \\
\hline $\begin{array}{l}\text { Advanced } \\
\text { human vs. } \\
\text { advanced non- } \\
\text { human }\end{array}$ & $\begin{array}{c}3.09 \\
(1.48)\end{array}$ & $\begin{array}{c}2.94 \\
(1.61)\end{array}$ & $\begin{array}{c}2.06 \\
(1.46)\end{array}$ & $25.68^{* * *}$ & .08 & -1.15 & -.09 & $-1.03^{* * *}$ & -.70 & $-0.88^{* * *}$ & -.58 \\
\hline $\begin{array}{l}\text { Advanced } \\
\text { human vs. basic } \\
\text { non-human }\end{array}$ & $\begin{array}{c}2.73 \\
(1.83)\end{array}$ & $\begin{array}{c}2.43 \\
(1.67)\end{array}$ & $\begin{array}{c}1.81 \\
(1.48)\end{array}$ & $15.2^{* * *}$ & .05 & -0.31 & -.18 & $-0.93^{* * *}$ & -.56 & $-0.62^{* * *}$ & -.39 \\
\hline
\end{tabular}




\begin{tabular}{|c|c|c|c|c|c|c|c|c|c|c|c|}
\hline $\begin{array}{l}\text { Basic human vs. } \\
\text { advanced non- } \\
\text { human }\end{array}$ & $\begin{array}{c}4.03 \\
(2.04)\end{array}$ & $\begin{array}{c}3.88 \\
(1.93)\end{array}$ & $\begin{array}{c}2.80 \\
(1.97)\end{array}$ & $21.67^{* * *}$ & .07 & -0.14 & -.07 & $-1.23^{* * *}$ & -.61 & $-1.08^{* * *}$ & -.55 \\
\hline $\begin{array}{l}\text { Basic human vs. } \\
\text { advanced human }\end{array}$ & $\begin{array}{c}4.65 \\
(1.89)\end{array}$ & $\begin{array}{c}4.71 \\
(1.74)\end{array}$ & $\begin{array}{c}4.65 \\
(1.68)\end{array}$ & 0.06 & $<.001$ & 0.05 & .03 & 0.002 & .001 & -0.05 & -.03 \\
\hline $\begin{array}{l}\text { Basic non- } \\
\text { human vs. } \\
\text { advanced non- } \\
\text { human }\end{array}$ & $\begin{array}{c}4.96 \\
(1.81)\end{array}$ & $\begin{array}{c}5.17 \\
(1.62)\end{array}$ & $\begin{array}{c}5.45 \\
(1.70)\end{array}$ & $3.86^{* *}$ & .01 & 0.20 & .12 & 0.49 & .28 & 0.28 & .17 \\
\hline
\end{tabular}

Table 3. Prioritization judgments $M(S D)$ of Study 2.1 stands for prioritizing the first individual, 4 for flipping a coin to decide whom to prioritize, and 7 for prioritizing the second individual. Rows represent the dilemmas in which two individuals are pitted against each other.

Columns represent the type of species the respective non-human is a member of, i.e., the three between-subjects conditions. ${ }^{*} p<.05 .{ }^{* * *} p<.01$. *** $p<.001$ 


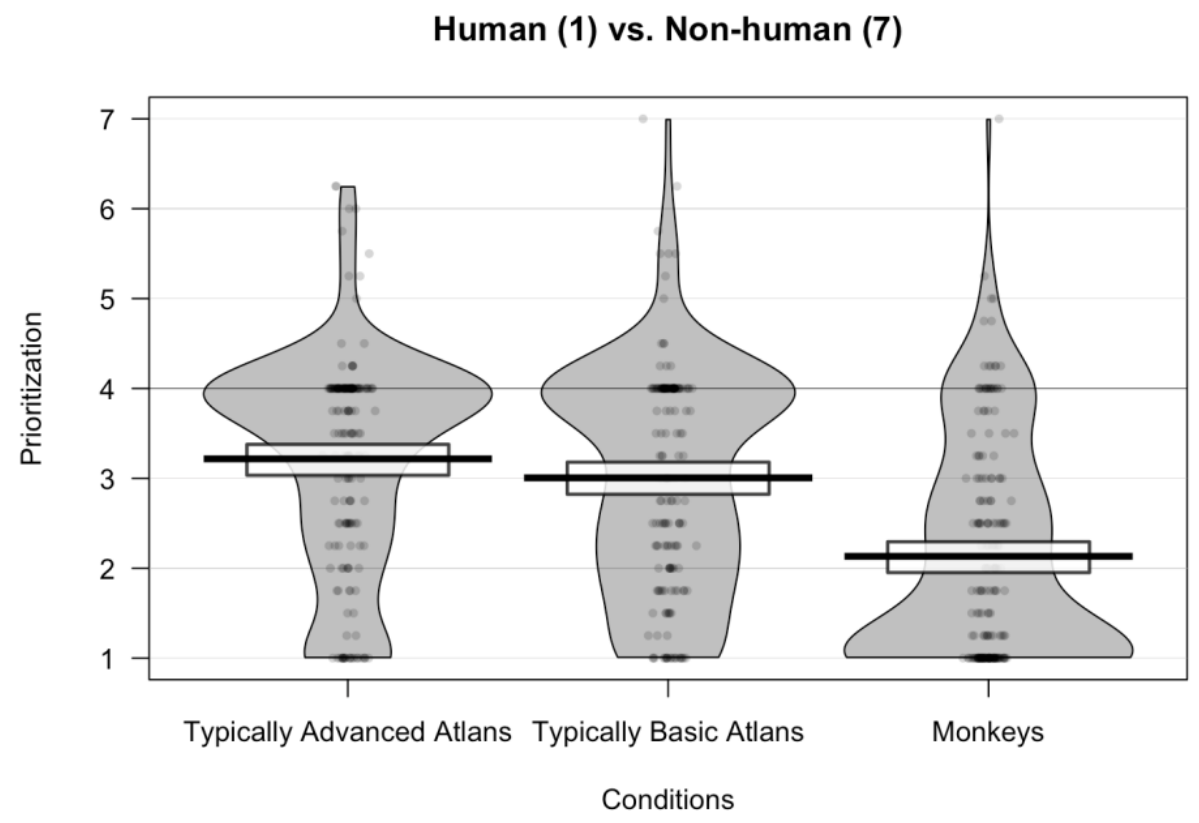

Figure 3. Many participants prioritized humans over non-humans. It made no difference whether the individual extraterrestrials (Atlans) were members of a typically mentally advanced or a typically mentally basic extraterrestrial species. The tendency to prioritize humans over extraterrestrials, however, was weaker than the tendency to prioritize humans over monkeys. For more detailed results of the individual dilemmas see Table 3 or the separate figures in the Appendix. (Study 2)

\section{Discussion}

In Study 2, we tested the species-typical mental capacity hypothesis - the view that people value humans more than animals because the typical human has advanced mental capacities, whereas the typical member of an animal species does not. The results speak against this hypothesis. First, many participants prioritized humans over typically mentally advanced extraterrestrials, contradicting what the species-typical mental capacity hypothesis predicts. Second, participants tended to treat members of typically mentally advanced and 
typically mentally basic extraterrestrials species equally, both when they were pitted against humans and against each other. This again contradicts the species-typical mental capacity Hypothesis.

We also found - in line with Studies 1a-b — that the individual's mental capacity does influence the moral status people attribute to it, even though species-membership has a much stronger influence.

A surprising finding was that people appear to value extraterrestrials more than monkeys: they were more inclined to prioritize humans over monkeys than to prioritize humans over extraterrestrials (including typically mentally basic extraterrestrials). We will investigate this finding further in Study 4.

A limitation of this study is that we did not ask participants to rate the mental capacity levels of all the different types of individuals on a slider-scale as we did in the previous studies. Instead, we relied on a comprehension check that only asked about the mental capacity level of the species-typical individual. It is possible, therefore, that some participants had incorrect assumptions about the mental capacity levels of some individuals.

\section{Study 3a: Species-Relativism vs Species-Absolutism}

In Studies 1a-b and 2 we tested whether people value humans more than non-humans because of their respective individual or species-typical mental capacity level. We found that these hypotheses cannot fully explain why people value humans given that many people give priority to humans over non-humans regardless of their respective individual mental capacities, and regardless of the typical mental capacity level of the non-human species. While individual mental capacity made some difference, it cannot explain the massive difference in perceived moral status between humans and non-humans. In other words, 
people likely exhibit speciesism. In Study 3a, we wanted to further investigate the source of speciesism. One hypothesis is that people think that humans should value other humans more, because we belong to the same species (species-relativism). Alternatively, it could be that people think that humans should be prioritized not because of shared species-membership, but merely because they are humans (species-absolutism).

To tease these two hypotheses apart we modify a factor which we have not varied in the previous studies, namely the species-membership of the would-be rescuer. In our previous studies, participants were asked how they themselves think they should decide in such a situation. This means that the rescuer was always a human being. In this study, by contrast, we ask participants how they think that rescuers belonging to different species should decide. Thus, the species-membership of the rescuer was manipulated. This allows us to tease apart the species-relativism and the species-absolutism hypotheses. The species-relativism hypothesis predicts that participants will think that the rescuer should always prioritize members of their own species over other individuals, including humans. By contrast, the species-absolutism hypothesis predicts that participants will think that all rescuers, irrespective of species-membership, should always prioritize humans over other individuals, including members of the rescuer's own species.

This study features cases involving relatively cognitively impaired humans and relatively intelligent chimpanzees with comparable mental abilities. Thus, the beings featured in this study are potentially realistic. The scenarios involve moral dilemmas in which one individual — either a chimpanzee or a human — must decide whether to help their fellow species members or members of the respective other species. Our hypothesis was that most participants believe that individuals should prioritize members of their own species (speciesrelativism), but that they believe this to a stronger extent for humans than for chimpanzees 
(species-absolutism). Our study was pre-registered at https://aspredicted.org/blind.php?x=7qe746.

\section{Methods}

Participants. We recruited 503 US American participants online via MTurk. They received \$0.6 in payment for their participation. 109 were excluded for failing either the attention or comprehension check, leaving a final sample of 394 people $\left(175\right.$ female; $M_{a g e}=$ 41.24, $\left.S D_{\text {age }}=12.64\right)$. Sample size was determined before any data analysis. We aimed to recruit 500 participants.

Materials and Procedure. The study had two between-subjects conditions: one where the rescuer was a human and one where the rescuer was a chimpanzee. Participants were told about a scenario involving scientists who study how relatively smart chimpanzees solve problems side by side with cognitively impaired humans of comparable ability. The participants were informed about the mental capacities of the humans and chimpanzees. They were said to have a number of abilities, including a basic sense of judging right from wrong. Next, participants were informed that due to an accident, some chimpanzees and humans had got locked inside the facility. They were told that the scientists could not reach the trapped individuals, and that the only way for them to get food was via one "rescuer" individual. Depending on the condition, the rescuer is a human or a chimpanzee. This rescuer needs to decide whether to give the food to humans or chimpanzees. Participants were asked what they think the rescuer should do, from an ethical perspective. They answered the same question twice. First, on a binary response scale $(0=$ to [the fellow species members], $1=$ to [the members of the other species]). Second, on a 7-point response scale (1 ='It's much more ethically right for the [rescuer] to give the food to the [fellow species members]', $4=$ Either action is equally ethically right for the [rescuer] to take, 7 ='It's much more ethically right 
for the [rescuer] to give the food to the [members of the other species]'). Next, participants responded to a comprehension check question that asked what the question was about (To make a prediction what an individual is most likely to do [6\% incorrectly selected this]; To make an ethical assessment of what an individual should do [78\% correctly selected this]; To judge whether a human or a chimpanzee is more moral [14\% incorrectly selected this]; To predict what an individual will think about different options [1\% incorrectly selected this]).

\section{Results}

First, we analyzed the binary variable without a middle option. We found that in both conditions the majority of participants believed individuals should prioritize members of ther own species over others. $79 \%$ of participants said the human rescuer should prioritize the humans over the chimpanzees $\left(\chi^{2}(1)=74.78, p<.001\right)$, while $60 \%$ participants said the chimpanzee rescuer should prioritize the chimpanzees over the humans $\left(\chi^{2}(1)=6.88, p=\right.$ $.01)$. This difference between the conditions was significant, $\chi^{2}(1)=15.31, p<.001$.

On the 7-point response scale, the average participant believed that the human rescuer should prioritize humans over chimpanzees $(M=2.90, S D=1.59)$. This was significantly below the mid-point $(4), t(225)=-10.4, p<.001, d=.69$. By contrast, the average participant believed that it was roughly equally right for the chimpanzee rescuer to either prioritize the chimpanzees or the humans $(M=3.85, S D=1.62)$. The mean judgment was not significantly above or below the mid-point, $t(167)=-1.19, p=.24, d=.09$. There was a significant difference between the two conditions (Figure 4), $t(355)=5.80, p<.001, d=.59$. Due to the large number of exclusions, we conducted the same analyses again without any exclusions. The pattern of results for all analyses remained the same (see Supplemenatary Materials). 
Own species (1) vs. Other species (7)

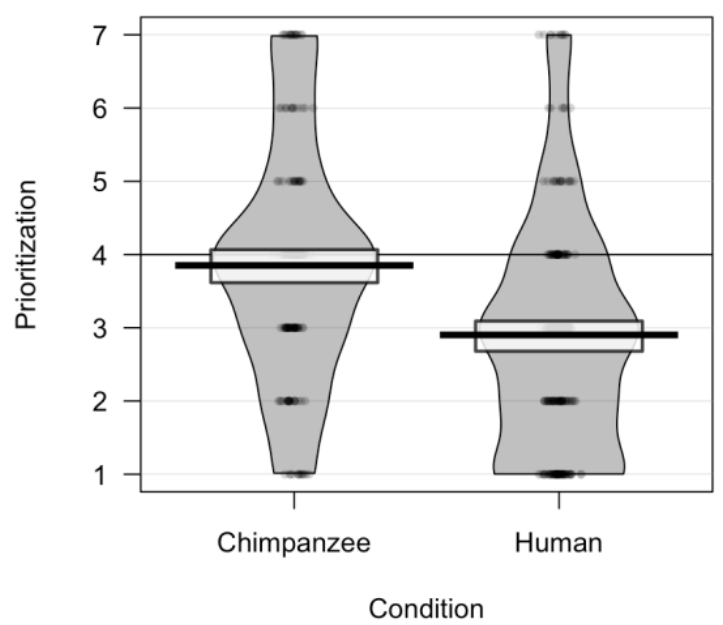

Figure 4. The average participant believed that a human should prioritize humans over chimpanzees but that it is equally ethically right for a chimpanzee to either help humans or chimpanzees. This was the case even though the described mental capacity level of the humans and chimpanzees was the same. In both conditions there were notable individual differences. (Study 3a)

\section{Discussion}

In Studies 1a-b, we found that while people do give greater moral priority to individuals with more advanced mental capacities over those with lower ones (individual mental capacity principle), this factor does not fully explain the massive moral priority that people give to humans over animals. We concluded that people likely prioritize humans over animals partly purely because of species-membership: they exhibit speciesism. In Study $3 \mathrm{a}$ we studied what underlies this phenomenon. We pitted two hypotheses against each other: species-relativism, the view that each species should prioritize their own, and pro-human species-absolutism, the view that everyone, including non-humans, should prioritize humans. We found that neither of these hypotheses on their own fully explained our findings. The 
species-relativism hypothesis found partial support in the fact that many participants believed that a chimpanzee rescuer should prioritize other chimpanzees over humans in cases where they only have a binary choice. However, species-relativism is also partly contradicted by the fact that participants had a stronger tendency to believe that humans should prioritize humans over chimpanzees of comparable mental capacities than to believe that chimpanzees should prioritize chimpanzees over humans.

How can these findings be reconciled? Our interpretation is that, as hypothesized in the introduction, people prioritize humans over non-humans for a multiplicity of reasons. People give some moral weight to individual mental capacity, while at the same time discriminate beings on the basis of mere species-membership, which appears to be partly captured by both species-relativism and species-absolutism—or more specifically in this case, pro-human species-absolutism. In the standard case where humans are pitted against animals with weaker mental capacities, all three factors apply, leading to the familiar massive priority given to humans over other animals. In this the scenario of this study, both speciesrelativism and pro-human species-absolutism appear to play a role.

\section{Study 3b: Species-Relativism with Different Non-Humans}

In Study 3a we showed that both species-relativism and pro-human speciesabsolutism likely are parts of the explanation of moral anthropocentrism. In Study 3b, we aimed to replicate this finding with a few changes. First, while in Study 3a all beings at stake had below-human mental capacities, in this study, all beings at stake had advanced humanlevel mental capacities. This means that, in contrast to Study 3a, we are again required to rely on partly unrealistic hypothetical scenarios. Second, instead of chimpanzees, we relied on hypothetical extraterrestrial species (referred to as "Atlans" and "Bredlans"). These changes 
allowed us to investigate what people believe a rescuer should do in cases involving two different types of non-human species with advanced human-level mental capacities.

Several of our hypotheses pertained to the species-relativism view-that people believe that individuals should prioritize members of their own species. First, we hypothesized that participants would be more likely to say that the Atlan rescuer should prioritize the Atlan over the Bredlan than that the human rescuer should do that. Second, we hypothesized that participants would be more likely to say that the human rescuer should prioritize the human over the Bredlan than that the Atlan rescuer should do that. Third, we hypothesized that participants would be more likely to say that the human rescuer should prioritize the human over the Atlan than that the Atlan rescuer should do that. These hypotheses left open the possibility of pro-human species-absolutism playing a role as well. For example, we hypothesized that pro-human species-absolutism would lead people to find it more important for a human rescuer prioritize a human over an Atlan than for an Atlan rescuer to prioritize an Atlan over a human. Finally, we hypothesized that religious people would be more likely to hold a pro-human species-absolutism because several religions hold the view that humans are a special species that is in an absolute sense more valuable than animals. To test this, we also measured religiosity. This study was pre-registered at https://osf.io/3nf9y/?view_only=2290e0e37c8a438a821b0b80a0c42829.

\section{Methods}

Participants. We recruited 225 US American participants online via MTurk. They received $\$ 0.6$ in payment for their participation. Eleven were excluded for failing at least one attention or comprehension check or for not completing the study, leaving a final sample of 
214 people $\left(100\right.$ female; $\left.M_{a g e}=40.65, S D_{a g e}=11.75\right)$. Sample size was determined before any data analysis. We aimed to recruit 220.

Materials and Procedure. The study had two between-subjects conditions, where the species of the rescuer was either human rescuer or extraterrestrial (again called 'Atlan'). Participants in each condition were presented with three moral dilemmas in randomized order. The three dilemmas pitted a human beneficiary against an Atlan beneficiary, a human beneficiary against a Bredlan beneficiary, and an Atlan beneficiary against a Bredlan beneficiary. The mental capacity levels of the beneficiaries were described as equally advanced for all three types of beings. Participants were asked to indicate which of the two individuals the rescuer should prioritize from an ethical standpoint on a 7-point scale $(1=$ Definitely A, $4=$ Flip a coin to decide, $7=$ Definitely $B$ ). All instructions were identical to those in Study 2, apart from the fact that we now asked the individuals what another individual should do (rather than what they themselves should do). After the main task, participants were presented with exploratory questions reported in the Supplementary Materials. Finally, they responded to the Centrality of Religiosity Scale (CRS; Huber \& Huber, 2012).

\section{Results}

First, there were proportionately more participants who believed that a human rescuer should prioritize a human over an Atlan than participants who believed that an Atlan rescuer should prioritize an Atlan over a human (Table 4; Figure 5), $\chi^{2}(1)=4.72, p=.03 .55 .8 \%$ of participants believed that a human should prioritize a human over an Atlan, while $43.2 \%$ believed the human should flip a coin. By contrast, only $40.1 \%$ of participants believed that an Atlan should prioritize an Atlan over a human, while $46.3 \%$ believed the Atlan should flip 
a coin. Next, we looked at the mean responses (Table 4). The average participant believed that a human should prioritize a human over an Atlan, $t(103)=9.40, p<.001, d=.92$, and that an Atlan should prioritize an Atlan over a human, $t(109)=3.50, p<.001, d=.33$. In line with our analysis above, the average participant was more likely to believe that a human should prioritize a human over an Atlan than to believe that an Atlan should prioritize an Atlan over a human, $t(206)=3.22, p=.002, d=.44$.

Second, there were proportionately more participants who believed that an Atlan rescuer should prioritize an Atlan over a Bredlan than participants who believed that a human rescuer should prioritize an Atlan over a Bredlan, $\chi^{2}(1)=49.73, p<.001 .45 .5 \%$ participants believed that an Atlan should prioritize an Atlan over a Bredlan, while $48.2 \%$ believed the human should flip a coin. By contrast, only $1.9 \%$ of participants believed that a human should prioritize an Atlan over a Bredlan, while 95.2\% believed the human should flip a coin. Next, we looked at the mean responses. The average participant believed that an Atlan should prioritize an Atlan over a Bredlan, $t(109)=5.77, p<.001, d=.55$, whereas the human should flip a coin, $t(103)=0.53, p=.60, d=.05$. In line with our analysis above, the average participant was more likely to believe that an Atlan should prioritize an Atlan over a Bredlan than that a human should prioritize an Atlan over a Bredlan, $t(125)=5.43, p<.001, d=.72$.

Third, there were proportionately more participants who believed that a human rescuer should prioritize a human over a Bredlan than participants who believed that an Atlan rescuer should prioritize a human over a Bredlan, $\chi^{2}(1)=20.37, p<.001 .48 .1 \%$ participants believed that a human should prioritize a human over a Bredlan, while $50.9 \%$ believed the human should flip a coin. By contrast, only $18.2 \%$ of participants believed that an Atlan should prioritize a human over a Bredlan, while the vast majority of $71.8 \%$ believed the Atlan should flip a coin. Next, we looked at the mean responses. The average participant believed 
that a human should prioritize a human over a Bredlan, $t(103)=8.18, p<.001, d=.80$, and that an Atlan should prioritize a human over a Bredlan, $t(109)=2.27, p=.03, d=.22$. In line with our analysis above, the average participant was more likely to believe that a human should prioritize a human over a Bredlan than that an Atlan should prioritize a human over a Bredlan, $t(211)=4.30, p<.001, d=.59$.

Against our hypothesis, there were no significant associations between religiosity (as measured by CRS as well as by stated religious affiliations such as Catholicism) and dilemma responses.

\section{Prioritize A Flip a coin Prioritize B M (SD)}

Human (A) vs Atlan (B)

$\begin{array}{lrrrr}\text { Human } & 55.8 \% & 43.2 \% & 1.0 \% & 2.85(1.25) \\ \text { Atlan } & 13.6 \% & 46.3 \% & 40.1 \% & 4.53(1.58)\end{array}$

Atlan (A) vs Bredlan (B)

$\begin{array}{lllll}\text { Human } & 2.9 \% & 95.2 \% & 1.9 \% & 3.98(0.37) \\ \text { Atlan } & 45.5 \% & 48.2 \% & 6.3 \% & 3.24(.39)\end{array}$

Human (A) vs Bredlan (B)

$\begin{array}{lrrrr}\text { Human } & 48.1 \% & 50.9 \% & 1.0 \% & 3.02(1.22) \\ \text { Atlan } & 18.2 \% & 71.8 \% & 10.0 \% & 3.74(1.22)\end{array}$

Table 4. Proportions of participants who believed that a human or Atlan rescuer should either save individual A, flip a coin to decide, or save individual B. The last column contains the means and standard deviations. (Study 3b) 

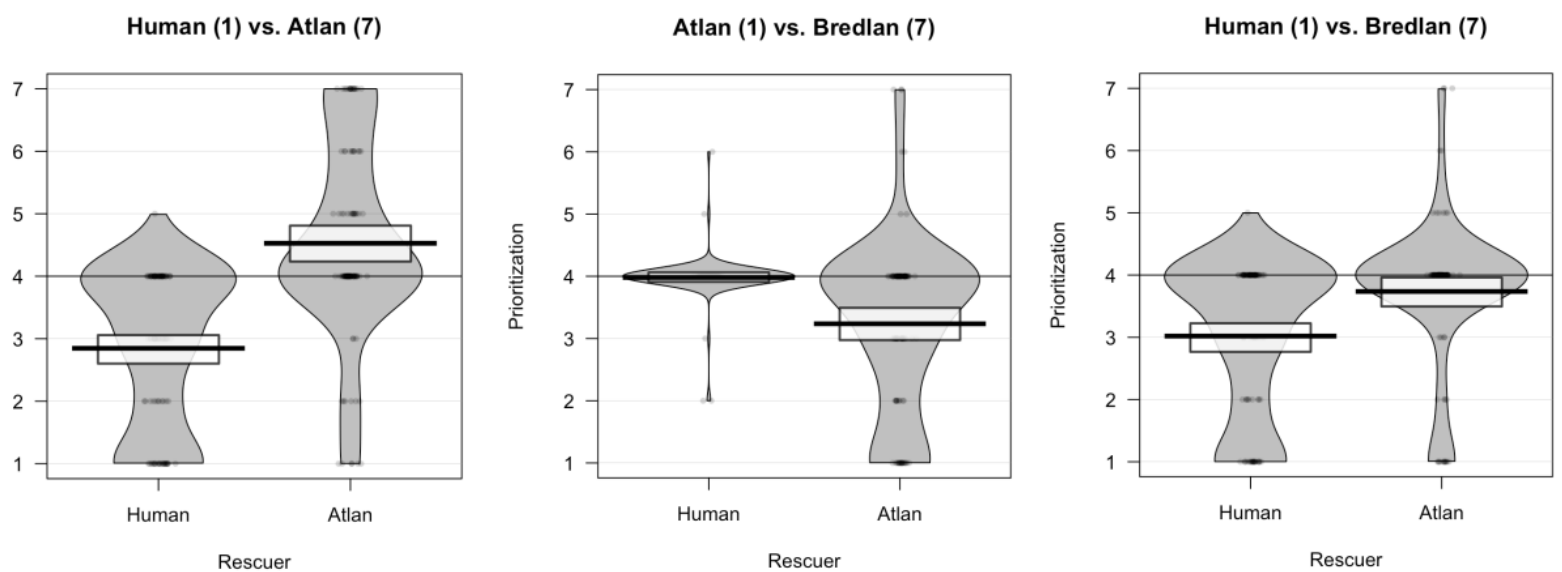

Figure 5. The modal response in all dilemmas was that the rescuer should flip a coin to decide which of the two individuals to save. However, many participants also believed that both humans and intelligent extraterrestrials should prioritize members of their own species over other individuals. And on average, there was an slightly increased overall tendency towards prioritizing humans. (Study 3b)

\section{Discussion}

The results of Study $3 b$ further confirm the existence of both species-relativism as well as species-absolutism. For example, most participants believed that humans should prioritize humans over non-humans. And similarly, a sizeable proportion of participants believed that intelligent extraterrestrials (Atlans) should prioritize members of their own species over other members of other species of intelligent extraterrestrials (Bredlans), and even over humans. But at the same time, there were proportionally more participants who believed that a human should prioritize another human over Atlans than participants who believed an Atlan should prioritize another Atlan over humans. Note, though, that only a minority of participants showed a pro-human species-absolutism tendency, whereas most did 
not. It's also noteworthy that the modal response in all cases was the belief that the rescuer should flip a coin to decide. For example, $72 \%$ of participants believed an Atlan should flip a coin to decide between prioritizing a human and a Bredlan. That is, many people were egalitarian between individuals of different species with similar advanced mental capacities.

Our hypothesis that religious people might be more likely to show pro-human speciesabsolutism—-given that some religious traditions portray humanity as having special cosmic importance-was not confirmed. Future research could explore in more detail whether moral attitudes towards animals differ across people with different religious beliefs. It is noteworthy that Christian views, such as the natural law tradition in Roman Catholicism, do not necessarily hold that humans are more valuable than members of other, equally rational, species.

\section{Study 4: Harming and Anti-Animal Species-Absolutism}

In all our studies so far, we presented participants with difficult prioritization decisions, where they chose between helping a human or helping a non-human. These are decisions where, in other contexts, partiality is often seen as justified. For instance, most people would find it acceptable to prioritize helping one's own family members over others (Petrinovich et al., 1993). In cases where one party would be actively harmed for the sake of the other party, partiality is likely to be more controversial. However, with respect to animals, people do consider it relatively permissible to actively harm and even systematically exploit animals to benefit humans as shown in the real-world context of consumption and medical experimentation as well as by recent psychological research (Caviola et al., 2020). We therefore wanted to investigate whether the factors that were found to drive moral 
anthropocentrism in the helping context also explain this permissive attitude to harm to animals.

This attitude cannot be entirely explained by the lower mental capacities of animals (the mental capacity principle) given that we do not accept such harm to humans with significant cognitive impairments. It is also unlikely to be fully explained by a combination of lower mental capacities and membership of a different species than humans (speciesrelativism), given that in Study 2 we found that people gave greater priority to extraterrestrials with basic mental capacities than to animals with the same capacities. We therefore hypothesized that a further factor must apply here - a specific bias against beings that are categorized as animals, over and above their specific or typical capacities - which we call "anti-animal species-absolutism". However, an alternative explanation of that finding is that people perceived the hypothetical extraterrestrials as having more human-like mental capacities, or as having more advanced mental capacities, than the monkeys. In Study 4 we therefore aimed to study whether people manifest such anti-animal species-absolutism, and whether it may explain permissive attitudes to harming animals. We hypothesized that participants, like in Study 2, would be more willing to not help a monkey than an Atlan with similar mental capacities, and that they would be more willing to actively harm a monkey than an Atlan in order to benefit a human. We further hypothesized that the difference in willingness to harm an animal compared to an Atlan would be greater than the difference in willingness to not help them. We assumed this based on the observation that harm towards and exploitation of animals in the real world is considered permissible to particularly strong extent. 


\section{Methods}

Participants. We recruited 271 US American participants online via MTurk. They received $\$ 0.6$ in payment for their participation. Twenty-two were excluded for failing at least one attention or comprehension check or for not completing the study, leaving a final sample of 249 people (109 female; $\left.M_{a g e}=42.50, S D_{a g e}=12.83\right)$. Sample size was determined before any data analysis. We aimed to recruit 270 participants.

Materials and Procedure. The study had a between-subjects 2 species (Atlan vs. monkey) x 2 type (help vs. harm) design. The vignettes were based on those of Study 2 . Participants were informed that while most typical Atlans (or monkeys) had basic mental capacities, a few special Atlans (or monkeys) had mentally advanced mental capacities. Next, they were presented with two moral dilemmas in randomized order. In one of the dilemmas, a mentally basic Atlan (or monkey) was pitted against a mentally basic human, and in the other dilemma a mentally advanced Atlan (or monkey) was pitted against a mentally advanced human. Depending on the condition, the dilemmas either involved a helping situation identical to the ones in the previous studies, or it involved a situation in which an Atlan (or monkey) had to be harmed in order to help a human. The harm dilemma read as follows: "Suppose you were in a situation in which the only way to help a human that has basic mental capacities is by actively harming an Atlan [a monkey] that has basic mental capacities." Participants were then asked to indicate what they should do from ethical standpoint on a 7-point scale (1 = Definitely NOT harm the Atlan [monkey], $4=$ Flip a coin to decide, 7 = Definitely harm the Atlan [monkey]).

After the main task, participants responded to three follow-up questions. First, they were asked to indicate on a scale from 1 to 100 how they would rate the mental capacities of a typical Atlan (or monkey) if a typical human was 100. Second, they were asked to indicate 
on a scale from 1 to 100 how similar a typical Atlan (or monkey) is to a human. Third, they were asked to indicate on a scale from 1 (Not at all) to 7 (Completely) to what extent they think a typical Atlan (or monkey) is an animal. These three questions were included to test whether any differences in the moral dilemma responses could be explained by either different perceptions of mental capacity levels, human-likeness or animal-likeness (our hypothesis).

\section{Results}

The response patterns in the basic and advanced mental capacities dilemmas were similar $($ rhelp $=.80$ and rharm $=.84)$. We, therefore, created an average score for the responses of the two dilemmas and conducted our analyses based on this.

$63.3 \%$ of participants prioritized saving the human over the Atlan but only $81.8 \%$ prioritized saving the human over the monkey, $\chi^{2}(1)=4.55, p=.03$. Only $35.1 \%$ were willing to harm the Atlan to save the human, whereas $57 \%$ were willing to harm the monkey to save the human, $\chi^{2}(1)=5.33, p=.02$. Note that in these analyses we created a binary variable which was either 1 (helping the human or harming the non-human) or 0 (flipping a coin or not helping the human / not harming the non-human).

Next, we looked at the mean responses by conducting 2x2 between-subjects ANOVA. This analysis yielded two main effects and no interaction (Figure 6). The average participant prioritized a human more over a monkey than over an Atlan and was more likely to harm a monkey than an Atlan to help a human, $F(1,245)=15.34, p<.001, \eta_{p}{ }^{2}=.06$. And the average participant was in general more likely to prioritize helping a human over a nonhuman than to actively harm a non-human to save a human, $F(1,245)=41.21, p<.001, \eta_{p}^{2}=$ .15 . There was no interaction between species and type, $F(1,245)=0.37, p=.55, \eta_{p}^{2}=.001$. 
The same pattern of results was found when ANOVAs were conducted on the responses of the two dilemmas individually (Table 5 for descriptive statistics).

\begin{tabular}{lllll}
\hline & \multicolumn{3}{l}{ Atlan } & \multicolumn{2}{l}{ Monkey } \\
\cline { 2 - 5 } & Harm & Help & Harm & Help \\
\cline { 2 - 5 } Basic & $4.23(2.05)$ & $2.65(1.56)$ & $3.17(1.97)$ & $2.14(1.50)$ \\
Advanced & $4.46(2.10)$ & $2.97(1.56)$ & $3.56(1.99)$ & $2.05(1.43)$ \\
Averaged & $4.34(2.00)$ & $2.81(1.45)$ & $3.36(1.88)$ & $2.09(1.42)$ \\
\hline
\end{tabular}

Table 5. Means and standard deviations of the responses in the moral dilemmas involving mentally basic and mentally advanced individuals (Study 4). Lower scores indicate greater willingness to help humans instead of non-humans and greater willingness to actively harm non-humans to save humans (reversed).

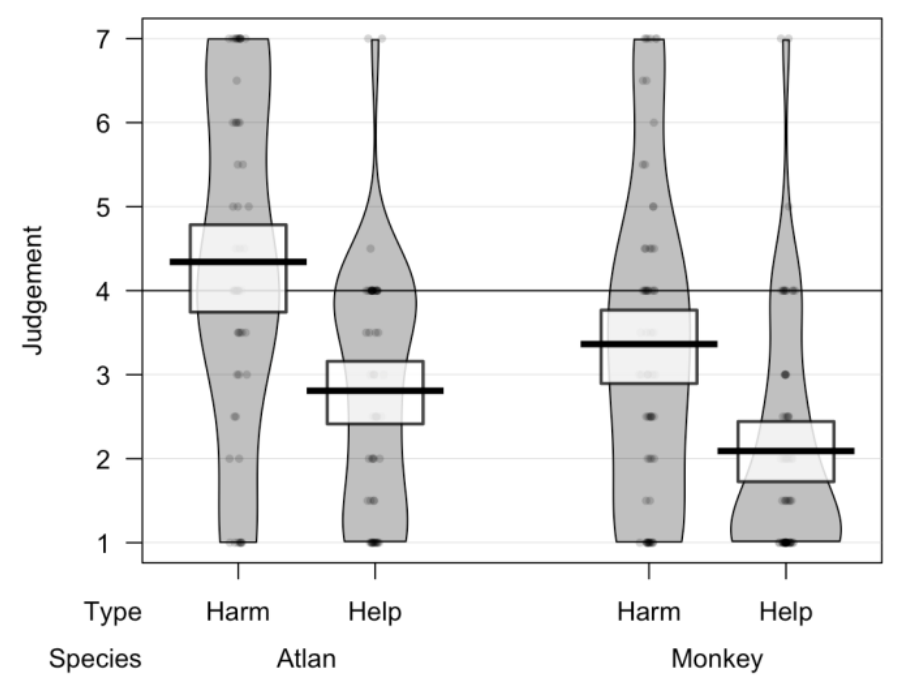


Figure 6. More participants prioritized the human over the monkey than over the Atlan. And more participants were willing to harm the monkey to save the human than to harm the Atlan to save the human. In the harm conditions 1 stands for Definitely harm the Atlan/monkey and 7 for Definitely NOT harm the Atlan/monkey (reversed). In the help conditions 1 stands for Definitely prioritize the human and 7 for Definitely prioritize the Atlan/monkey. 4 stands for Flip a coin to decide in all conditions (Study 4).

There were no significant differences in the level of mental capacities they believed typical Atlans $(M=43.25, S D=24.50)$ had compared to typical monkeys $(M=39.56, S D=$ 22.39), $t(236.5)=1.23, p=0.22, d=.16$. Similarly, there were no significant differences in the extent to which participants perceived Atlans $(M=44.71, S D=26.35)$ and monkeys $(M=$ $45.60, S D=24.88)$ to be human-like, $t(239)=-0.27, p=0.79, d=-.03$. However, participants perceived a typical monkey $(M=6.50, S D=1.04)$ as an animal to a greater extent than they did a typical Atlan $(M=4.28, S D=1.85), t(178)=11.48, p<.001, d=-1.50$.

\section{Discussion}

In line with our main hypothesis, this study suggests that many people appear to have a specific bias against animals, which we call anti-animal species-absolutism. More participants prioritized the humans over the monkeys than the Atlans, and more participants were willing to harm the monkeys than to harm the Atlans to benefit humans. This was the case even though the mental capacities of both the monkeys and Atlans were described in identical terms, and even though both were perceived as having similar human-like levels of mental capacities. Participants exhibited similar levels of priority to Atlans over monkeys to in help and harm scenarios. This suggests that there may be only a difference of degree 
between the helping judgments investigated in the previous studies and parallel judgments about harming.

The tendency to prioritize Atlans over monkeys is puzzling. We found that people perceived the monkeys as more animal-like than an Atlan. Therefore, one possible conclusion is that some people have a specific bias against animals or animal-like beings in particular. Let us look at how such an anti-animal species-absolutism fits with our framework. We originally hypothesized that there are two forms of speciesism: species-relativism and species-absolutism. We have now observed that there two components of species-absolutism: pro-human (Studies 3a-c) and anti-animal species-absolutism (Studies 2 and 4). Just like prohuman species-absolutism, anti-animal species-absolutism is an absolute bias. People do not value extraterrestrial Atlans more than monkeys because of a special relationship between helpers and beneficiaries, but rather because they seem to find animals less valuable in an absolute sense.

We wish to emphasize that the anti-animal species-absolutism hypothesis is an exploratory component of this project. It only came to our attention after discovering signs of such a potential effect in Study 2. But neither Study 2 nor Study 4 provide definitive proof for the existence of anti-animal species-absolutism, since it remains possible that the effect might be explained by other factors that we did not account for.

It is worth mentioning that there was an asymmetry between the harm and help scenarios we used. While in the help scenarios both options involved an action (to help one but not another one), in the harm scenarios one option involved an action and the other an omission. If in the harm scenarios instead both options involved either harming a human or a non-human, people would presumably prioritize the human much more strongly than in our study. 


\section{General Discussion}

Humans regard themselves as having priority over animals, an attitude that manifests itself in numerous policies, practices, and choices, ranging from consumption of animal meat and animal experimentations to zoos and animals performing in circuses. Our aim was to identify the factors that underlie this attitude and present a first attempt of a framework for moral anthropocentrism, which future research can study in-depth. We found that moral anthropocentrism is underpinned not by a single factor but by several distinct ones. While perceived differences in individual mental capacity can partly explain why people value humans more than animals, they cannot fully explain it. Instead, our studies suggest that the central driver of moral anthropocentrism is speciesism, which can itself be divided into further sub-components.

\section{The Mental Capacity Principle}

If the only reason why people value humans more was their more advanced mental capacities, we should expect them to value non-humans with similarly or more advanced mental capacities as much as humans (individual mental capacity principle). However, that was not the case. In Studies 1a-b we found that many participants prioritized humans over animals even if animals had equal (or even stronger) mental capacities. However, while manipulating individual mental capacity could never flip the priority from humans to animals, it did contribute to the strength to which participants prioritized humans over animals: the more advanced the mental capacities of the animals were, the weaker the extent to which participants prioritized humans over animals. Many participants also prioritized mentally more advanced non-humans over mentally less advanced non-humans of the same 
species. These findings extend previous research that found associations between an animal's perceived mental capacity level and their perceived moral status (e.g., Bastian et al., 2012; Caviola et al., 2019, 2020). And crucially, these findings demonstrate that individual mental capacity does play a role in explaining moral anthropocentrism even if it cannot explain it in full.

Our findings rule out one important candidate for explaining moral anthropocentrism, the species-typical mental capacity principle: the view that the moral status of an individual is based on the mental capacity level of the typical member of the species. Moral philosophers often invoke this view to explain why so-called 'marginal cases', such as severely cognitively impaired humans, should be given full moral status and treated radically differently than animals, even when they do not possess greater mental capacities (see e.g., Cohen, 1986; Finnis, 1997; Scanlon, 1998). However, in Study 2 we found that the typical mental capacity level of a species had no significant effect on participants' judgments in the moral dilemmas. Of course, these results do not directly show that the species-typical mental capacity principle is mistaken as an ethical claim. But they do reveal a radical disconnect between a popular defense of moral anthropocentrism by some philosophers and the factors that actually underpin the moral thinking of lay people.

\section{The Sub-Factors of Speciesism}

In line with previous research (Caviola et al., 2019), we found that people are speciesists - that they prioritize humans over animals simply because they are humans. We deepened and extended that research through identifying three forms of speciesism: speciesrelativism, pro-human species-absolutism, and anti-animal species-absolutism. 
First, Studies 3a-b showed that many people follow a species-relativist principle, according to which individuals of all species should prioritize members of their own species over others. For example, many participants judged that mentally advanced extraterrestrial beings should prioritize members of their own species over mentally advanced members of other species, including humans. Participants were less likely, however, to accept that monkeys or chimpanzees should prioritize themselves over more advanced species. This could be because they believe that mentally advanced beings are more valuable than mentally basic ones, which in this case counteracts species-relativism. Alternatively, it is possible that people are less inclined to apply species-relativism to mentally basic species or simply to animals due to anti-animal species-absolutism.

Species-relativism appears to function in a way akin to partiality towards one's family. People generally prioritize members of their family over others (Petrinovich et al., 1993) while also holding that other people should similarly prioritize members of their families (Pugh et al., 2013). Patriotism is another example. species-relativism is analogous to a US American saying "America first" and at the same time considering it legitimate for a French person to say "France first". Partiality to one's family members or compatriots are widely seen as morally permissible and, in some contexts, even obligatory (Keller, 2013; Miller, 2005). It has even been found that a significant number of people would prioritize their own pet over a foreign tourist, suggesting that partiality towards family (in this case, a pet) can even overturn moral anthropocentrism (Topolski et al., 2013). Importantly, prioritization based in partiality need not reflect a difference in moral status or value. Parents may prioritize their children over others without believing that their children have a higher moral status. 
In addition to species-relativism we found evidence of tendencies that we call speciesabsolutism, since they apply irrespective of the relationship between the helper and beneficiary. The first species-absolutist tendency we have identified is pro-human speciesabsolutism. Most notably, participants were more likely to say that a human should prioritize a human over an Atlan than they were to say that an Atlan should prioritize an Atlan over a human. pro-human species-absolutism differs from the "America first" patriotism example above but would be more like American Exceptionalism—-seeing the US nationals as superior to all other nations in an absolute sense. It is possible that pro-human species-absolutism is primarily implicit and that most people would not endorse it under reflection (e.g., when the bias is made explicit). Whether people — and which people — really believe humans are more valuable qua humans needs to be investigated further. A further question is how people would justify such a bias, given that it may operate even when the non-humans had identical or stronger cognitive capacities.

The second species-absolutist tendency we have identified is anti-animal speciesabsolutism. In Study 4 we found that participants were less likely to help and more likely to harm monkeys than equally mentally capable Atlans. Similarly, in Study 2, we found that participants treated monkeys worse than typically mentally basic extraterrestrials. We believe that the evidence for the existence of anti-animal species-absolutism is clearly weaker than the evidence for the other key factors we have explored in this paper. And we consider these results as exploratory and in need for further investigation. One possible explanation of the effect is that people value terrestrial animals less than other, equally mentally capable, nonhumans simply because they perceive them as more animal-like. An alternative explanation could be that people tend to believe that humans feel a special moral obligation towards extraterrestrial species which they don't feel to the same extent towards terrestrial animals 
with equal mental capacities. Yet another possibility is that the effect we found is not real and that it is explained by a confounder that we weren't able to sufficiently control for. For example, it could be that it is more difficult for people to imagine highly intelligent monkeys than highly intelligent extraterrestrials. More research is required to explore the anti-animal species-absolutism hypothesis more rigorously, but we hope to have provided an interesting first step in such a line of research.

\section{Relations Among the Factors of Moral Anthropocentrism}

Speciesism and the individual mental capacity principle both contribute to moral anthropocentrism, as we have seen. Let us now look more precisely at the causal relations between these factors (Figure 7). When someone attributes a lower moral value to one being (an animal) than to another (a human) because of speciesism, there are two judgments involved: the perception that the two beings belong to different species, and the moral judgment that one of the species is morally more valuable than the other. It is the combination of these two judgments that contribute to moral anthropocentrism (1). Similarly, when someone attributes a lower moral value to one being than to another because of the individual mental capacity principle, there are two judgments involved: the perception that one of the beings is mentally more advanced than the other, and the moral judgment that it therefore is morally more valuable (2).

These two links (1 and 2) are direct relations between the main factors we have demonstrated and moral anthropocentrism. However, there are also indirect causal links between these factors. First, there is an indirect link between speciesism and perceptions of relative mental capacities of humans and animals: speciesism causes people to attribute lower mental capacities to animals (3). This tendency has been referred to as de-mentalization and has been demonstrated in previous research that showed that people attribute lower mental 
capacities to food animals compared with non-food animals (e.g., Bastian et al., 2012). Further, it has been found that speciesism correlates with attributions of lower mental capacities to animals (Caviola et al., 2018).

Second, there is an indirect link between speciesism and the individual mental capacity principle. People sometimes appear to apply the individual mental capacity principle selectively to animals and not to humans (4). In Studies $1 \mathrm{a}-\mathrm{b}$ and 2 we found that participants valued animals with higher mental capacities more than animals with lower mental capacities. At the same time, however, participants in Studies 1a-b did not to the same extent value humans with higher mental capacities more than humans with lower mental capacities. This tendency to selectively apply the individual mental capacity principle more to animals than to humans could itself be seen as speciesist—as pointed out in the philosophical literature (Singer, 1975, chapter 1). Thus, while our research shows that many people apply the individual mental capacity principle to animals, more research is required to understand whether and when they also apply it to humans.

According to our framework, then, speciesism reduces the perceived moral status of an animal in multiple ways: both directly and indirectly. This suggests that speciesism plays a more central role in explaining moral anthropocentrism than one might think at first glance. Future research needs to verify and refine this framework. In particular, the relative strength of each factor remains unclear. It's also unclear which — if any — of these factors people exclusively endorse when asked why they value humans more than animals. 


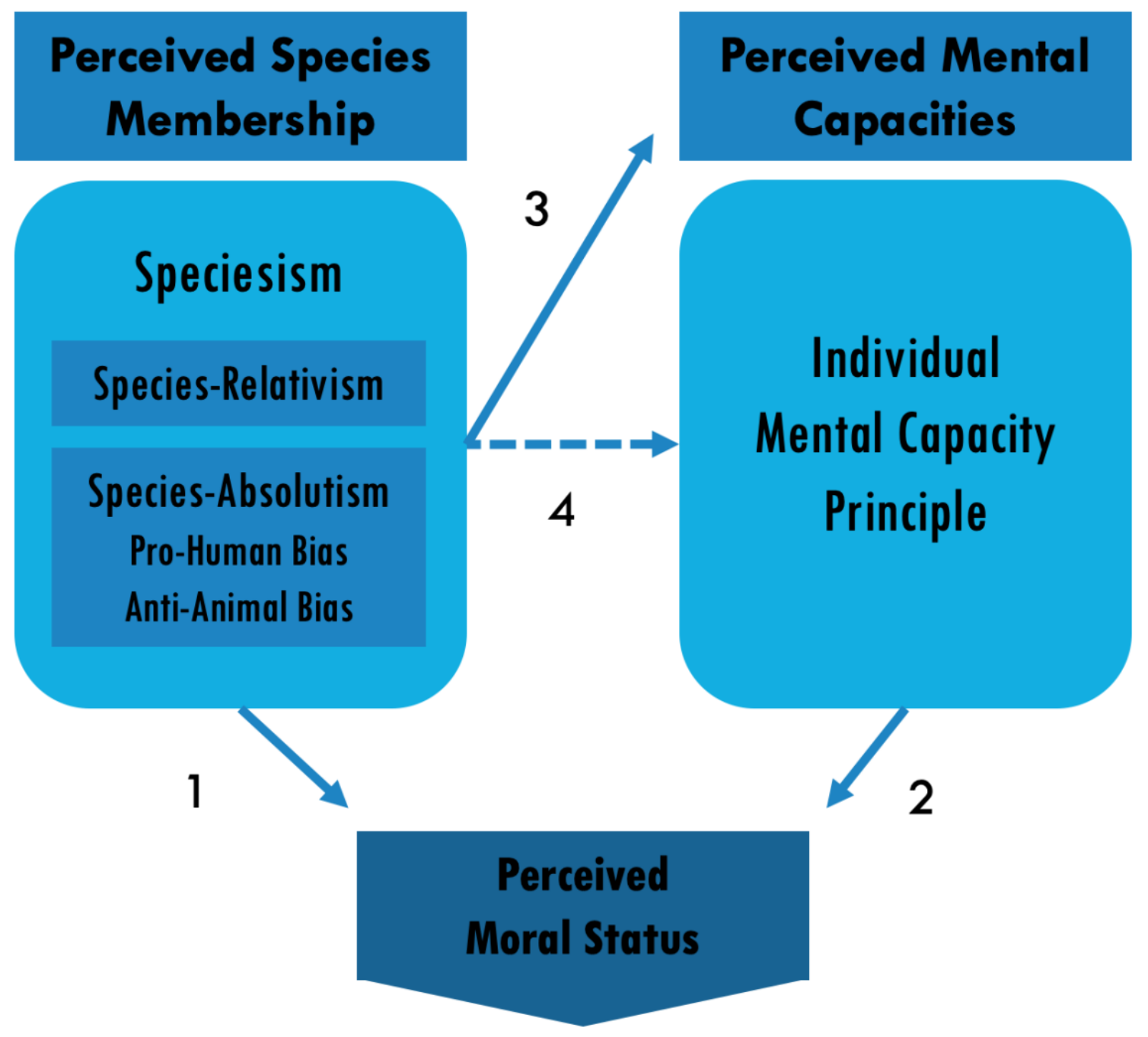

Figure 7. A Framework for moral anthropocentrism. One reason (1) people perceive nonhumans to have low moral status is because of mere species-membership, i.e., speciesism Another reason (2) is the fact that people attribute lower moral status to beings that have low perceived mental capacities, i.e., individual mental capacity principle. Furthermore, there are two indirect paths from speciesism to moral anthropocentrism. First (3), speciesism leads people to ascribe lower mental capacities to animals. Second (4), people selectively apply the individual mental capacity principle more to animals than to humans, a phenomenon which may itself be driven by speciesism. 


\section{The Distal Sources of Moral Anthropocentrism}

So far, we have discussed how the factors of moral anthropocentrism are related to each other. We now turn to briefly discuss what ultimate factors may explain moral anthropocentrism, though at present there is little evidence that directly bears on this question. However, evolutionary considerations suggest a preliminary, if inevitably speculative, account of the ultimate sources of moral anthropocentrism. Such an explanation could also shed light on the role of the sub-factors of speciesism.

There is extensive evidence that people categorize individuals into different groups (cf. Tajfel et al., 1971), identify with their own group (Hornsey, 2008; Tajfel et al., 1979), and prioritize members of their ingroup over members of their outgroup (Balliet et al., 2014; Crimston et al., 2016; Fu et al., 2012; Sherif, 1961; Yamagishi \& Kiyonari, 2000; for bounded generalized reciprocity theory, cf. Yamagishi \& Mifune, 2008). Ingroup favoritism is expressed in many different contexts. People have, for example, a tendency to favor others who share their ethnicity, nationality, religion, or political affiliation. (Rand et al., 2009; Whitt \& Wilson, 2007). It has been argued that ingroup favoritism is an innate tendency since it can promote safety and help to encourage mutual cooperation among ingroup members (Gaertner \& Insko, 2000). It seems, therefore, that there are good reasons to assume that speciesism is a form of ingroup favoritism analogous to ingroup favoritism amongst human groups.

While typical human ingroups would be far smaller than humanity itself, our similarity to other humans would be salient in contexts where a choice needs to be made between a human and a non-human. Since the differences between humans and animals are perceived as vast — in terms of biology, physical appearance, mental capacities, and 
behavior - and thus the boundaries between the groups so wide and clear, one would expect ingroup favoritism between humans and animals to be particularly strong. Indeed, research suggests that perceived similarity with outgroup members can reduce ingroup favoritism —as long as they are seen as non-threatening (Henderson-King et al., 1997). Similarly, it has been shown that people have more positive reactions towards animals that are perceived as biologically, physically, mentally, or behaviorally more similar to humans than animals that are dissimilar (Burghardt \& Herzog, 1989; Kellert \& Berry, 1980)

The prioritization of humans over others could, thus, in significant part be an extension of familiar ways in which we prioritize ourselves and those linked to us, regardless of other considerations (e.g., to do with mental capacity). Spelled out explicitly, as a 'universalizable' moral principle, this would generate the idea of species-relativism. But perhaps less explicitly and consciously, it is also reflected in pro-human species-absolutisma degree of priority given to humans simply because they are human, independently of the actual relationship between helper and potential beneficiary. In line with this, recent research found that even young children have a tendency to prioritize humans over animals, although it is much weaker than in adults (Wilks et al., 2021)

In addition, people also have a desire to signal that they are fair moral agents, a disposition that is evolutionarily adaptive because it increases the chances of others engaging in mutual cooperation with us (DeScioli \& Kurzban, 2013; Tooby \& Cosmides, 2010). Processes of prioritization of the kind investigated here provide a context in which such fairness can be signaled. Accepting that others are permitted to prioritize their own group (in this case, a non-human species) can be a way to signal such fairness while at the same time having a justification to prioritize our own when we can. At the same time, since our expected audience is invariably human, treating humans more important in a more absolute 
sense can still send an attractive signal - and possibly even more so in cultural contexts where contemporary ideas of moral equality are less dominant.

Crucially, though, people only have to signal their positive moral character traits to individuals who are themselves capable of engaging in moral reasoning and in potential mutual cooperation, i.e., to mentally advanced individuals. There is no gain in justifying to mentally basic individuals why they are being deprioritized because they would not understand the signal. This, in addition to similarity to self, can explain why people value mentally basic individuals less (in an absolute sense) than mentally advanced individuals (viz. the individual mental capacity principle). However, people are still capable of empathetic responses to the welfare of non-humans with lower mental capacities, and thereby still perceive them as having some moral status. Moreover, attitudes to individuals with lower mental capacities (typically, non-human animals) still send a valuable signal to other individuals with higher mental capacities — someone engaged in gratuitous cruelty to animals indicates low affective empathy and reduced inhibition to harm, dispositions that would importantly bear on their interactions with other humans.

Finally, it appears that people also categorize animals not just as non-human (and thus belonging to a distant outgroup), or as having lower mental capacities, but positively as animals. How can the anti-animal species-absolutism be explained? People might be accustomed to treating animals as profoundly morally inferior and may associate being an animal with low moral status, whereas they are not accustomed to interacting with extraterrestrials. Moreover, a specific prejudice against animal-like beings could have been evolutionarily adaptive. Automatic classification of entities in one's environment as animals — as opposed to inanimate objects, plants or, of course, humans — was obviously evolutionarily adaptive. Realistic conflict theory assumes that conflict of interests between 
groups can lead to negative attitudes towards outgroup members (Sherif, 1961). Clearly, there are many conflicts of interests between humans and animals. Historically, animals either preyed on humans, or were preyed on by them (cf., Kasperbauer, 2017). Therefore, it is plausible that humans have developed a specific bias against animals that they might not have towards other kinds of non-humans.

\section{Limitations and Future Research}

While Studies 1a and 3a involved scenarios with humans and animals with a fairly realistic range of mental capacities, other studies partly involved merely hypothetical beings such as intelligent animals and extra-terrestrials. Given that participants never encountered such beings and that the scenarios involved choices that are distant from familiar moral situations, questions may be raised about drawing conclusions about everyday attitudes to humans and animals from responses to such unusual situations. We concede that this is a methodological limitation of some of our studies. However, we wish to note that most of the key effects were also robustly observed in the studies involving more realistic humans and animals (Studies 1a and 3c). And although some of our studies relied on unrealistic scenarios, our comprehension checks (in particular in Studies 1a-b and 4) suggest that participants regarded the intelligent animals as having the same mental capacities as the humans. Moreover, while participants of course never encountered intelligent chimpanzees or extraterrestrials, such beings are widely portrayed in fiction and film, often in contexts that explicitly or implicitly involve moral choice. Participants thus arguably have the capacity both to vividly imagine such beings and to assign to them comparative moral status. In any event, in our view it is not possible to experimentally investigate many of our research questions without using such scenarios. A great deal of current research into moral judgments 
employs scenarios - such as those involving runaway trolleys - that are arguably as unrealistic as the scenarios used here. We concede, though, that further research tying some of the factors we have identified to behavior in more realistic contexts could both reinforce our findings and demonstrate their relevance to actual moral choices.

Future research could also help clarify whether the discovered factors play a significant causal role in driving behavior towards animals or rather reflect post-hoc justifications of such behavior. This is in particular important since all our findings depend on self-reports in an online context. It cannot be ruled out that people's responses in our studies reflect more their beliefs about what is socially desirable than the reactions they would have if they were to encounter individuals of different species and with different mental capacities in the real world. In addition, future research could also uncover the psychological mechanisms that underlie the factors we have identified here.

In our studies the only animal species participants were presented with were monkeys and chimpanzees. However, previous research has shown that people perceive some animals to be morally more important than others, e.g., dogs compared to pigs (Caviola et al., 2019; Caviola \& Capraro, 2020). Future research could investigate how the different factors underlying moral anthropocentrism play out when different animals are compared with each other, such as animals that are usually categorized as food, experimental subjects or pets. It is also possible that further factors underlying moral anthropocentrism will be discovered that go beyond the ones we have identified, e.g., biases against specific categories of animals, such as pests or dangerous animals (Piazza, Landy, \& Goodwin, 2014)

\section{Conclusion}


We found that people prioritize humans over non-humans for multiple reasons. First, many people value animals less than humans in part because individual animals have lower mental capacities than individual humans (individual mental capacity principle). However, this alone cannot fully explain moral anthropocentrism, since most people continue to prioritize humans over animals even in cases when the animals have the same or even higher mental capacities the humans. Instead, the most central factor explaining anthropocentrism is speciesism, which itself has multiple sub-factors. We found that many people believe that individuals should prioritize members of their owns species over others (species-relativism), and that some people consider humans to be of superior value in an absolute sense (prohuman species-absolutism). We also found evidence suggesting that, in addition, there might also be a specific bias against animals, a bias that people don't manifest towards non-humans with equivalent mental capacities (anti-animal species-absolutism).

As we show, these four factors can come apart in hypothetical scenarios. However, since all of them apply to all actual animals, it is not surprising that moral anthropocentrism is such a robust and pervasive attitude. Attempts to show that we routinely underestimate the mental capacities of animals, or that we are linked with them by relations of similarity or fellowship, can influence only some aspects of moral anthropocentrism. Moreover, while differences in mental capacity, and partiality to group members, can arguably justify prioritizing humans in at least some contexts, species-absolutism-regarding individual humans as absolutely morally more important simply in virtue of being humans (pro-human species-absolutism), and individual animals as absolutely morally inferior simply in virtue of being animals (anti-animal species-absolutism)—clearly seems like a bias. Our studies thus demonstrate that moral anthropocentrism is partly driven by prejudice. 


\section{References}

Amiot, C. E., \& Bastian, B. (2015). Toward a psychology of human-animal relations. Psychological Bulletin, 141(1), 6-47. https://doi.org/10.1037/a0038147

Balliet, D., Wu, J., \& De Dreu, C. K. W. (2014). Ingroup favoritism in cooperation: A metaanalysis. Psychological Bulletin, 140(6), 1556-1581. https://doi.org/10.1037/a0037737

Bandura, A. (1999). Moral disengagement in the perpetration of inhumanities. Personality and Social Psychology Review, 3(3), 193-209.

Bastian, B., Loughnan, S., Haslam, N., \& Radke, H. (2012). Don't Mind Meat? The Denial of Mind to Animals Used for Human Consumption. Personality \& Social Psychology Bulletin, 38, 247-256. https://doi.org/10.1177/0146167211424291

Bratanova, B., Loughnan, S., \& Bastian, B. (2011). The effect of categorization as food on the perceived moral standing of animals. Appetite, 57(1), 193-196.

Burghardt, G. M., \& Herzog, H. A. (1989). Animals, evolution and ethics. Perceptions of Animals in American Culture, 129-151.

Caviola, L., \& Capraro, V. (2020). Liking but devaluing animals: Emotional and deliberative paths to speciesism. Social Psychological and Personality Science.

Caviola, L., Everett, J. A. C., \& Faber, N. S. (2019). The moral standing of animals: Towards a psychology of speciesism. Journal of Personality and Social Psychology. https://doi.org/10.1037/pspp0000182 
Caviola, L., Kahane, G., Everett, J., Teperman, E., Savulescu, J., \& Faber, N. S. (2020). Utilitarianism for Animals, Kantianism for People? Harming animals and humans for the greater good. Journal of Experimental Psychology: General.

Cohen, C. (1986). The case for the use of animals in biomedical research. N. Engl. J. Med., 315(14), 865-870.

Crimston, D., Bain, P. G., Hornsey, M. J., \& Bastian, B. (2016). Moral expansiveness: Examining variability in the extension of the moral world. Journal of Personality and Social Psychology, 111(4), 636.

Douglas, T. (2013). Human enhancement and supra-personal moral status. Philosophical Studies, 162(3), 473-497.

DeScioli, P., \& Kurzban, R. (2013). A solution to the mysteries of morality. Psychological Bulletin, 139(2), 477.

Dhont, K., Hodson, G., Costello, K., \& MacInnis, C. C. (2014). Social dominance orientation connects prejudicial human-human and human-animal relations. Personality and Individual Differences, 61, 105-108.

Dhont, K., Hodson, G., \& Leite, A. C. (2016). Common Ideological Roots of Speciesism and Generalized Ethnic Prejudice: The Social Dominance Human-Animal Relations Model (SD-HARM). European Journal of Personality, 30(6), 507-522. https://doi.org/10.1002/per.2069

Dhont, K., Hodson, G., Loughnan, S., \& Amiot, C. E. (2019). Rethinking human-animal relations: The critical role of social psychology. SAGE Publications Sage UK: London, England.

Finnis, J. (1997). The fragile case for euthanasia: A reply to John Harris. Euthanasia Examined: Ethical, Clinical and Legal Perspectives, 46-55. 
Fu, F., Tarnita, C. E., Christakis, N. A., Wang, L., Rand, D. G., \& Nowak, M. A. (2012). Evolution of in-group favoritism. Scientific Reports, 2, 460.

Gaertner, L., \& Insko, C. A. (2000). Intergroup discrimination in the minimal group paradigm: Categorization, reciprocation, or fear? Journal of Personality and Social Psychology, 79(1), 77.

Goodwin, G. P. (2015). Experimental approaches to moral standing. Philosophy Compass, 10, 914-926.

Henderson-King, E., Henderson-King, D., Zhermer, N., Posokhova, S., \& Chiker, V. (1997). In-group favoritism and perceived similarity: A look at Russians' perceptions in the post-Soviet era. Personality and Social Psychology Bulletin, 23(10), 1013-1021.

Hornsey, M. J. (2008). Social Identity Theory and Self-categorization Theory: A Historical Review. Social and Personality Psychology Compass, 2(1), 204-222. https://doi.org/10.1111/j.1751-9004.2007.00066.x

Huber, S., \& Huber, O. W. (2012). The centrality of religiosity scale (CRS). Religions, 3(3), $710-724$.

Jaworska, A., \& Tannenbaum, J. (2014). Person-rearing relationships as a key to higher moral status. Ethics, 124(2), 242-271.

Kagan, S. (2019). How to Count Animals, more or less. Oxford University Press.

Kahane, G., Everett, J. A. C., Earp, B. D., Caviola, L., Faber, N., Crockett, M. J., \& Savulescu, J. (2017). Beyond sacrificial harm: A two dimensional model of utilitarian decision-making.

Kant, I. (1797/2017). The metaphysics of morals, Gregor M (trans). Cambridge University Press, Cambridge. 
Kasperbauer, T. J. (2017). Subhuman: The Moral Psychology of Human Attitudes to Animals. Oxford University Press.

Keller, S. (2013). Partiality (Vol. 40). Princeton University Press.

Kellert, S. R., \& Berry, J. K. (1980). Knowledge, Affection and Basic Attitudes Toward Animals in American Society. Phase III.

Loughnan, S., Haslam, N., \& Bastian, B. (2010). The role of meat consumption in the denial of moral status and mind to meat animals. Appetite, 55(1), 156-159. https://doi.org/10.1016/j.appet.2010.05.043

Miller, D. (2005). Reasonable partiality towards compatriots. Ethical Theory and Moral Practice, 8(1-2), 63-81.

Opotow, S. (1993). Animals and the scope of justice. Journal of Social Issues, 49(1), 71-85.

Petrinovich, L., O’neill, P., \& Jorgensen, M. (1993). An empirical study of moral intuitions: Toward an evolutionary ethics. Journal of Personality and Social Psychology, 64(3), 467.

Piazza, J., Landy, J. F., \& Goodwin, G. P. (2014). Cruel nature: Harmfulness as an important, overlooked dimension in judgments of moral standing. Cognition, 131(1), 108-124.

Plous, S. (1993). Psychological mechanisms in the human use of animals. Journal of Social Issues, 49(1), 11-52.

Pugh, J., Kahane, G., \& Savulescu, J. (2013). Cohen’s conservatism and human enhancement. The Journal of Ethics, 17(4), 331-354.

Rand, D. G., Pfeiffer, T., Dreber, A., Sheketoff, R. W., Wernerfelt, N. C., \& Benkler, Y. (2009). Dynamic remodeling of in-group bias during the 2008 presidential election. Proceedings of the National Academy of Sciences, 106(15), 6187-6191. 
Rottman, J., \& Kelemen, D. (2012). Aliens behaving badly: Children's acquisition of novel purity-based morals. Cognition, 124(3), 356-360.

Scanlon, T. (1998). What We Owe to Each Other. Harvard University Press.

Sherif, M. (1961). Intergroup conflict and cooperation: The Robbers Cave experiment (Vol. 10). University Book Exchange Norman, OK.

Sidanius, J., \& Pratto, F. (1999). Social Dominance by Jim Sidanius. Cambridge University Press.

Singer, P. (1975). Animal liberation: A new ethic for our treatment of animals. HarperCollins.

Singer, P. (1979). Practical Ethics (2 edition). Cambridge University Press.

Starmans, C., \& Friedman, O. (2016). If I am free, you can't own me: Autonomy makes entities less ownable. Cognition, 148, 145-153. https://doi.org/10.1016/j.cognition.2015.11.001

Sytsma, J., \& Machery, E. (2012). The Two Sources of Moral Standing. Review of Philosophy and Psychology, 3(3), 303-324.

Tajfel, H., Billig, M. G., Bundy, R. P., \& Flament, C. (1971). Social categorization and intergroup behaviour. European Journal of Social Psychology, 1(2), 149-178.

Tajfel, H., Turner, J. C., Austin, W. G., \& Worchel, S. (1979). An integrative theory of intergroup conflict. Organizational Identity: A Reader, 56-65.

Tooby, J., \& Cosmides, L. (2010). Groups in mind: The coalitional roots of war and morality. Human Morality and Sociality: Evolutionary and Comparative Perspectives, 91-234.

Topolski, R., Weaver, J. N., Martin, Z., \& McCoy, J. (2013). Choosing between the emotional dog and the rational pal: A moral dilemma with a tail. Anthrozoös, 26(2), $253-263$. 
Whitt, S., \& Wilson, R. K. (2007). The dictator game, fairness and ethnicity in postwar Bosnia. American Journal of Political Science, 51(3), 655-668.

Wilks, M., Caviola, L., Kahane, G., \& Bloom, P. (2020). Children prioritize humans over animals less than adults do.

Williams, B. (2009). The human prejudice. In Peter Singer Under Fire: The Moral Iconoclast Faces His Critics. Open Court.

Yamagishi, T., \& Kiyonari, T. (2000). The Group as the Container of Generalized Reciprocity. Social Psychology Quarterly, 63(2), 116-132. JSTOR. https://doi.org/10.2307/2695887

Yamagishi, T., \& Mifune, N. (2008). Does shared group membership promote altruism? Fear, greed, and reputation. Rationality and Society, 20(1), 5-30. 